\title{
COHERENT RINGS, FP-INJECTIVE MODULES, DUALIZING COMPLEXES, AND COVARIANT SERRE-GROTHENDIECK DUALITY
}

\author{
LEONID POSITSELSKI
}

\begin{abstract}
For a left coherent ring $A$ with every left ideal having a countable set of generators, we show that the coderived category of left $A$-modules is compactly generated by the bounded derived category of finitely presented left $A$-modules (reproducing a particular case of a recent result of Št'ovíček with our methods). Furthermore, we present the definition of a dualizing complex of fp-injective modules over a pair of noncommutative coherent rings $A$ and $B$, and construct an equivalence between the coderived category of $A$-modules and the contraderived category of $B$-modules. Finally, we define the notion of a relative dualizing complex of bimodules for a pair of noncommutative ring homomorphisms $A \longrightarrow R$ and $B \longrightarrow S$, and obtain an equivalence between the $R / A$-semicoderived category of $R$-modules and the $S / B$-semicontraderived category of $S$-modules. For a homomorphism of commutative rings $A \longrightarrow R$, we also construct a tensor structure on the $R / A$-semicoderived category of $R$-modules. A vision of semi-infinite algebraic geometry is discussed in the introduction.
\end{abstract}

\section{Contents}

Introduction 1

1. Fp-Injective and Fp-Projective Modules 4

2. Coderived Category of Modules over a Coherent Ring 6

3. Dualizing Complexes and Contravariant Duality 10

4. Covariant Duality Theorem 14

5. Semiderived Categories and Relative Dualizing Complexes 17

6. The Semitensor Product 23

$\begin{array}{ll}\text { References } & 28\end{array}$

\section{INTRODUCTION}

0.1. The philosophy of semi-infinite homological algebra, as elaborated in the book [14], tells that semi-infinite homology and cohomology theories are naturally assigned to mathematical objects "of semi-infinite nature", meaning objects that can be viewed as extending in both a "positive" and a "negative" direction with some "zero position" in between, perhaps defined up to a finite movement. In application 
to algebraic geometry, one thinks of a "semi-infinite algebraic variety" as an ind-proalgebraic variety or an ind-scheme of ind-infinite type, with the scheme or pro-variety variables forming the "negative direction" and the ind-variety variables belonging to the "positive" one. Thus the simplest example of a semi-infinite algebraic variety is the affine/vector space of formal Laurent power series $k((z))$ over a ground field $k$, and many more geometrically complicated examples are supposed to be constructed using the field structure of the Laurent power series.

More specifically, experience seems to suggest that the "positive" variables have to be "grouped together" in some sense, forming a well-defined "positive subalgebra" object in the "semi-infinite" algebra of functions or operators, like the subalgebra $z k[[z]] d / d z$ in the Lie algebra $k((z)) d / d z$ of vector fields on the formal circle. In the context of algebraic geometry, this points to a morphism of ind-schemes or ind-stacks $\pi: \mathfrak{Y} \longrightarrow \mathfrak{X}$ with, approximately, the following properties:

(I) $\mathfrak{Y}$ is a large and complicated ind-scheme or ind-stack;

(II) $\mathfrak{X}$ is built up in a complicated way from affine schemes of rather small size: something like an ind-Noetherian ind-scheme or an ind-Noetherian ind-stack with a dualizing complex;

(III) the morphism $\mathfrak{Y} \longrightarrow \mathfrak{X}$ is locally well-behaved: one would probably want it to be at least flat, or perhaps "very flat" in the sense of [18, Section 1.7];

(IV) the fibers of the morphism $\mathfrak{Y} \longrightarrow \mathfrak{X}$ are built up in a simple way from large affine pieces: so they might be arbibrary affine schemes, or quasi-compact semi-separated schemes, or perhaps some kind of "weakly proregular formal schemes" in the sense of $[27,19]$.

For example, the surjective linear map of topological vector spaces $k((z)) \longrightarrow$ $k((z)) / k[[z]]$ can be viewed naturally as a morphism of ind-schemes satisfying the conditions (I-IV). The discrete quotient space $k((z)) / k[[z]]$ is the set of $k$-points of an ind-scheme of ind-finite type over $k$, while the fibers, isomorphic to $k[[z]]$, are the sets of $k$-points of affine schemes of infinite type.

0.2. In the algebraic formalism of [14], the main starting object is a semialgebra $\mathcal{S}$, that is an associative algebraic structure "mixing algebra and coalgebra variables". The "positively indexed" variables form a coalgebra $\mathcal{C}$; the semialgebra $\mathcal{S}$ is an algebra object in the category of bicomodules over $\mathcal{C}$. The key structures in the categorical formalism are the semiderived categories of semimodules and semicontramodules over $\mathcal{C}$; these are mixtures of the co/contraderived categories "in the direction of $\mathcal{C}$ " and the conventional derived categories "in the direction of $\mathcal{S}$ relative to $\mathcal{C}$ ".

In the geometric situation described above, the purpose of having a morphism of ind-schemes or ind-stacks $\mathfrak{Y} \longrightarrow \mathfrak{X}$ is to consider the semiderived category of quasicoherent torsion sheaves or contraherent cosheaves of contramodules on $\mathfrak{Y}$ relative to $\mathfrak{X}$, which means "the co- or contraderived category along $\mathfrak{X}$ and the conventional derived category along the fibers". The "semi-infinite algebraic geometry" formalism would then feature a "geometric semimodule-semicontramodule correspondence", i. e., a triangulated equivalence between the two semiderived (or, if one wishes, the semicoderived and the semicontraderived) categories. 
In addition, one expects to have a "semi-infinite quasi-coherent Tor functor", or the double-sided derived functor of semitensor product of quasi-coherent torsion sheaves on $\mathfrak{Y}$. This means a mixture of the cotensor product [17, Section B.2.5] of quasi-coherent torsion sheaves along the ind-scheme/ind-stack $\mathfrak{X}$ with its dualizing complex and the conventional tensor product of quasi-coherent sheaves along the fibers. The derived semitensor product functor should provide a tensor structure on the semiderived category of quasi-coherent torsion sheaves, and the pull-back of the dualizing complex $\pi^{*} \mathcal{D}_{\mathfrak{X}}^{\bullet}$ should be the unit object of this tensor structure. One would also expect to have a double-sided derived functor of semihomomorphisms from quasi-coherent torsion sheaves to contraherent cosheaves of contramodules on $\mathfrak{Y}$, transformed by the derived semico-semicontra correspondence into the conventional right derived quasi-coherent internal $\mathcal{H}$ om.

0.3. The aim of the present paper is to work out a couple of small pieces in the above big picture. First of all, we attempt to show that the Noetherianness condition in (II) can be weakened to the coherence condition. The definition of a dualizing complex over a commutative coherent ring, or a pair of noncommutative ones, is elaborated for this purpose. On a more technical level, we demonstrate the usefulness of the notion of an fp-injective module over a coherent ring. Secondly, we introduce the definition of a relative dualizing complex and obtain an equivalence between the semicoderived and the semicontraderived categories of modules in the simplest geometric situation of a morphism of affine schemes $\mathfrak{Y} \longrightarrow \mathfrak{X}$. In addition, we construct the derived semitensor product functor in this situation, defining a tensor structure on the semiderived category of modules.

Notice that the case a quasi-compact semi-separated scheme $\mathfrak{Y}$ over a point $\mathfrak{X}=*$ has been already considered in [18, Section 4.6] and the case of a Noetherian scheme $\mathfrak{Y}$ over $\mathfrak{X}=*$, in [18, Theorem 5.8.1]. The case of a weakly proregular (e. g., Noetherian) affine formal scheme $\mathfrak{Y}$ over a point $\mathfrak{X}=*$ is clarified in the paper [19]. The situation of a (semi-separated or non-semi-separated) Noetherian scheme $\mathfrak{Y}=\mathfrak{X}$ with a dualizing complex has been considered in [18, Section 5.7 and Theorem 5.8.2]. The case of a semi-separated Noetherian stack $\mathfrak{Y}=\mathfrak{X}$ with a dualizing complex has been worked out in [18, Section B.4], and the case of an ind-affine ind-Noetherian ind-scheme $\mathfrak{Y}=\mathfrak{X}$ with a dualizing complex, in $[18$, Section D.2]. (The reader can find an overview of these results in the recent presentation [20].) The present paper adds an item or two to this list.

Acknowledgements. The mathematical content of this paper was conceived in Moscow and subsequently worked out in Haifa and in Brno. I am grateful to Henning Krause who told me about fp-injective modules during a workshop in Moscow in September 2011. I would like to thank Jan Št'ovíček for sending me his preprint [22] and Amnon Yekutieli for helpful discussions. The author was supported in part by RFBR grants in Moscow, by a fellowship from the Lady Davis Foundation at the Technion, and by the Grant agency of the Czech Republic under the grant P201/12/G028 at Masaryk University in Brno. The author's research is supported by the Israel Science Foundation grant \# 446/15 at the University of Haifa. 


\section{Fr-Injective And Fp-Projective Modules}

Hereditary complete cotorsion theories in abelian and exact categories [23, 5, 3], [1, Section 1.1], starting with the flat cotorsion theory in the category of modules over an associative ring and the very flat cotorsion theory in the category of modules over a commutative ring, and continuing with numerous others, play an important role in the theory of contraherent cosheaves [18].

The theory of fp-injective and fp-projective modules is one of the classical examples of complete cotorsion theories [24, Definition 3.3 and Theorem 3.4(2)], [10]. From our point of view, its importance in the study of modules over coherent rings lies in the fact that the class of fp-injective modules, while often not differing very much homologically from the narrower class of injective ones, is at the same time closed under infinite direct sums, and in fact, even under filtered inductive limits [21]. Thus the use of fp-injective modules allows to work with many coherent rings in the ways otherwise applicable to Noetherian rings only.

This section contains preliminary material, and the proofs are sketchy. Filling in the details is left to the reader.

Given an associative ring $A$, we denote by $A$-mod the abelian category of left $A$-modules and by mod- $A$ the abelian category of right $A$-modules. A left $A$-module $M$ is said to be finitely presented if it can be presented as the cokernel of a morphism of finitely generated free left $A$-modules. Clearly, the cokernel of a morphism from a finitely generated left $A$-module to a finitely presented one is finitely presented; an extension of finitely presented left $A$-modules is finitely presented.

Lemma 1.1. The kernel of a surjective morphism from a finitely generated module to a finitely presented one is finitely generated.

A ring $A$ is called left coherent if any finitely generated submodule of a finitely presented left $A$-module is finitely presented, or equivalently, if any finitely generated left ideal in $A$ is finitely presented as a left $A$-module. Whenever $A$ is a left coherent ring, the full subcategory $A-\bmod _{\mathrm{fp}}$ of finitely presented left $A$-modules is closed under the kernels, cokernels, and extensions in $A-\bmod$; so $A-$ mod $_{\mathrm{fp}}$ is an abelian category and its embedding $A-\bmod _{\mathrm{fp}} \longrightarrow A$-mod is an exact functor.

Lemma 1.2. Let $A$ be a left coherent ring, and let $C^{\bullet}$ be a bounded above complex of left $A$-modules whose cohomology modules $H^{n}\left(C^{\bullet}\right)$ are finitely presented over $A$. Then there exists a bounded above complex of finitely generated free left $A$-modules $F^{\bullet}$ together with a quasi-isomorphism of complexes of $A$-modules $F^{\bullet} \longrightarrow C^{\bullet}$.

Corollary 1.3. For any left coherent ring $A$, the triangulated functors between the derived categories of bounded and bounded above complexes $\mathrm{D}^{\mathrm{b}}\left(A-\bmod _{\mathrm{fp}}\right) \longrightarrow$ $\mathrm{D}^{\mathrm{b}}(A-\bmod )$ and $\mathrm{D}^{-}\left(A-\bmod _{\mathrm{fp}}\right) \longrightarrow \mathrm{D}^{-}(A-\bmod )$ induced by the embedding of abelian categories $A-\bmod _{\mathrm{fp}} \longrightarrow A$-mod are fully faithful. Their essential images coincide with the full subcategories $\mathrm{D}_{\mathrm{fp}}^{\mathrm{b}}\left(A\right.$-mod) and $\mathrm{D}_{\mathrm{fp}}^{-}(A$-mod $)$ of complexes with finitely presented cohomology modules in $\mathrm{D}^{\mathrm{b}}(A-\bmod )$ and $\mathrm{D}^{-}(A-\bmod )$. 
Let $A$ be a left coherent ring. A left $A$-module $J$ is said to be $f p$-injective [21] if the functor $\operatorname{Hom}_{A}(-, J)$ takes short exact sequences of finitely presented left $A$-modules to short exact sequences of abelian groups, or equivalently, if $\operatorname{Ext}_{A}^{1}(M, J)=0$ for any finitely presented left $A$-module $M$, or if $\operatorname{Ext}_{A}^{i}(M, J)=0$ for all finitely presented $M$ and all $i>0$. All injective modules are fp-injective. The class of fp-injective left modules over left coherent ring $A$ is closed under extensions, cokernels of injective morphisms, infinite direct sums and products, and filtered inductive limits. So, in particular, the full subcategory $A$ - $\bmod _{\mathrm{fpi}}$ of fp-injective left $A$-modules inherits the exact category structure of the abelian category $A$-mod.

The next definition and the related assertions, including the rest of this section and also Lemma 2.5(b) below, are never really used in the proofs of the main results of this paper. They are presented here for the sake of completeness of the exposition, and in the belief that the related techniques will find their uses in the future development of semi-infinite algebraic geometry.

A left $A$-module $P$ is said to be $f p$-projective $[24,10]$ if the functor $\operatorname{Hom}_{A}(P,-)$ takes short exact sequences of fp-injective left $A$-modules to short exact sequences of abelian groups, or equivalently, if $\operatorname{Ext}_{A}^{1}(P, J)=0$ for any fp-injective left $A$-module $J$, or if $\operatorname{Ext}_{A}^{i}(P, J)=0$ for all fp-injective $J$ and all $i>0$. All projective modules and all finitely presented modules are fp-projective. The class of fp-projective left modules over a left coherent ring $A$ is closed under extensions, kernels of surjective morphisms, and infinite direct sums. So the full subcategory $A-\bmod _{\mathrm{fpp}}$ of fp-projective left $A$-modules inherits the exact category structure of the abelian category $A$-mod.

Moreover, the class of fp-projective left $A$-modules is closed under transfinitely iterated extensions in the following sense ("of inductive limit"). A left $A$-module $P$ is said to be a transfinitely iterated extension of left $A$-modules $M_{\alpha}$ if there exist a wellordering of the set of indices $\{\alpha\}$ and an increasing filtration $F_{\alpha} P$ of the $A$-module $P$ by its $A$-submodules such that one has $\bigcup_{\alpha} F_{\alpha} P=P$ and for every index $\alpha$ the quotient module $F_{\alpha} P / \bigcup_{\beta<\alpha} F_{\beta} P$ is isomorphic to $M_{\alpha}$. The following result $[23,24]$ tells that there are "enough" fp-injective and fp-projective left $A$-modules.

Theorem 1.4. (a) Any left A-module can be embedded into an fp-injective left A-module in such a way that the quotient module is fp-projective.

(b) Any left A-module is the quotient module of some fp-projective left A-module by its fp-injective submodule.

The fp-projective modules in both parts of Theorem 1.4 are constructed as certain transfinitely iterated extensions of finitely presented modules. Hence it follows from (the proof of) part (b) that a left $A$-module $P$ is fp-projective if and only if it is a direct summand of a transfinitely iterated extension of finitely presented left $A$-modules.

Lemma 1.5. Let $A$ be a left coherent ring. Then any finitely generated submodule of an fp-projective left $A$-module is finitely presented.

Proof. It suffices to show that any finitely generated submodule $N \subset P$ of a transfinitely iterated extension $(P, F)$ of finitely presented left $A$-modules $M_{\alpha}$ is finitely presented. Let $\alpha_{0}$ be the minimal index $\alpha$ such that $N$ is contained in $F_{\alpha} P$ (since $N$ 
is finitely generated, such indices $\alpha$ exist). The quotient module $N / N \cap \bigcup_{\beta<\alpha} F_{\beta} P$ is a finitely generated submodule of a finitely presented left $A$-module $M_{\alpha}$, and consequently, also a finitely presented $A$-module. By Lemma 1.1 , the $A$-module $N \cap \bigcup_{\beta<\alpha} F_{\beta} P$ is finitely generated; and the assumption of induction in the ordinal $\{\alpha\}$ tells that it is finitely presented. Now the $A$-module $N$ is finitely presented as an extension of two finitely presented $A$-modules.

Lemma 1.6. Let $A$ be a left coherent ring, $P^{\bullet}$ be a complex of fp-projective left $A$-modules, and $J^{\bullet}$ be a complex of fp-injective left $A$-modules. Then whenever either the complex $P^{\bullet}$ is bounded above, or the complex $J^{\bullet}$ is bounded below, the Hom complex $\operatorname{Hom}_{A}\left(P^{\bullet}, J^{\bullet}\right)$ computes the groups $\operatorname{Hom}_{\mathrm{D}(A-\bmod )}\left(P^{\bullet}, J^{\bullet}[*]\right)$.

Proof. One notices that the complex $\operatorname{Hom}_{A}\left(P^{\bullet}, J^{\bullet}\right)$ is acyclic whenever either the complex $P^{\bullet}$ is a bounded above complex of projective $A$-modules and the complex $J^{\bullet}$ is acyclic, or the complex $P^{\bullet}$ is acyclic and the complex $J^{\bullet}$ is a bounded below complex of injective $A$-modules. Therefore, the complex $\operatorname{Hom}_{A}\left(P^{\bullet}, J^{\bullet}\right)$ computes the groups $\operatorname{Hom}_{\mathrm{D}(A \text {-mod })}\left(P^{\bullet}, J^{\bullet}[*]\right)$ whenever either $P^{\bullet}$ is a bounded above complex of projective $A$-modules, or $J^{\bullet}$ is a bounded below complex of injective $A$-modules.

Furthermore, the complex $\operatorname{Hom}_{A}\left(P^{\bullet}, J^{\bullet}\right)$ is acyclic whenever either the complex $P^{\bullet}$ is an acyclic bounded above complex of fp-projective left $A$-modules and $J^{\bullet}$ is a complex of fp-injective left $A$-modules, or $P^{\bullet}$ is a complex of fp-projective left $A$-modules and $J^{\bullet}$ is a bounded below acyclic complex of fp-injective left $A$-modules. Since any bounded above complex of $A$-modules is the target of a quasi-isomorphism from a bounded above complex of projective $A$-modules, and any bounded below complex of $A$-modules is the source of a quasi-isomorphism into a bounded below complex of injective $A$-modules, the desired assertions follow.

\section{Coderived Category of Modules over a Coherent Ring}

This section is our take on [9, Conjecture 5.9]. Notice that this conjecture of Krause's is already resolved (proven in the coherent and disproven in the noncoherent case) by Št'ovíček in [22, Theorem 6.12, Corollary 6.13, and Example 6.15]. The more elementary approach below is based on the techniques of working with derived categories of the second kind developed in [17] and formulated in the form convenient for us here in [18, Appendix A], instead of the set-theoretic methods of [22].

Given an additive category $E$, we denote by $\operatorname{Hot}(\mathrm{E})$ the homotopy category of (unbounded complexes over) E. We refer to [18, Section A.1] for the definitions of the coderived category $\mathrm{D}^{\mathrm{co}}(\mathrm{E})$ and the contraderived category $\mathrm{D}^{\mathrm{ctr}}(\mathrm{E})$ of an exact category $E$ with exact functors of infinite direct sum or infinite product, respectively. A slightly different definition of such categories was suggested by Becker in [1, Proposition 1.3.6]; it is also used in [22]. The definitions in [1] have the advantage of working well for the category of modules over an arbitrary ring (and also CDG-modules over an arbitrary CDG-ring). Our definitions have the advantage of being more explicit and applicable to abelian/exact categories of quite general nature. 
Proposition 2.1. Let $\mathrm{E}$ be an exact category with exact functors of infinite direct sum, and let $\mathrm{J} \subset \mathrm{E}$ be a full subcategory closed under infinite direct sums. Assume that the full subcategory $\mathrm{J}$ is closed under extensions in $\mathrm{E}$, and endow it with the induced exact category structure. Assume further that $\mathrm{J}$ is closed under the passages to the cokernels of admissible monomorphisms in $\mathrm{E}$, and that any object of $\mathrm{E}$ is the source of an admissible monomorphism into an object of $\mathrm{J}$. Then the triangulated functor $\mathrm{D}^{\mathrm{co}}(\mathrm{J}) \longrightarrow \mathrm{D}^{\mathrm{co}}(\mathrm{E})$ induced by the embedding of exact categories $\mathrm{J} \longrightarrow \mathrm{E}$ is an equivalence of triangulated categories.

Proof. This is the assertion dual to [18, Proposition A.3.1(b)].

Theorem 2.2. Let $A$ be a left coherent ring. Then the triangulated functor $\mathrm{D}^{\mathrm{co}}\left(A-\bmod _{\mathrm{fpi}}\right) \longrightarrow \mathrm{D}^{\mathrm{co}}(A-\bmod )$ induced by the embedding $A-\bmod _{\mathrm{fpi}} \longrightarrow A$-mod of the exact category of fp-injective A-modules into the abelian category of arbitrary A-modules is an equivalence of triangulated categories.

Proof. This is a particular case of Proposition 2.1. Similarly one can prove that the coderived category $\mathrm{D}^{\mathrm{co}}(A$-mod) of left CDG-modules over a CDG-ring $(A, d, h)$ with a left graded coherent underlying graded ring $A$ is equivalent to the coderived category $\mathrm{D}^{\mathrm{co}}\left(A-\bmod _{\mathrm{fpi}}\right)$ of left CDG-modules with fp-injective underlying graded modules. (Cf. the similar assertion about the contraderived categories of flat and arbitrary CDG-modules over CDG-rings with coherent underlying graded rings in [17, Remark 1.5].)

According to [4, Théorème 7.10], the projective dimension of a flat module over an associative ring of the cardinality $\aleph_{n}$ cannot exceed $n+1$. The following result is simpler, though sounds somewhat similar.

Proposition 2.3. Let $A$ be a left coherent ring such that any left ideal in $A$ admits a set of generators of the cardinality not exceeding $\aleph_{n}$, where $n$ is an integer. Then the injective dimension of any fp-injective left $A$-module is not greater than $n+1$.

Proof. By Baer's criterion, a left $A$-module $K$ is injective whenever $\operatorname{Ext}_{A}^{1}(A / I, K)=0$ for all left ideals $I \subset A$. Hence it suffices to prove that $\operatorname{Ext}_{A}^{n+2}(A / I, J)=0$ for all left ideals $I$ and all fp-injective left $A$-modules $J$. Any left ideal $I \subset A$ is the inductive limit of the filtered inductive system of its finitely generated subideals $I_{\alpha} \subset I \subset A$, and the quotient module $A / I$ is a filtered inductive limit of the quotient modules $A / I_{\alpha}$. Furthermore, for any filtered inductive system of left modules $L_{\alpha}$ and a left module $M$ over an associative ring $A$ there is a spectral sequence

$$
E_{2}^{p, q}=\lim _{\alpha}^{p} \operatorname{Ext}_{A}^{q}\left(L_{\alpha}, M\right) \Longrightarrow \operatorname{Ext}_{A}^{p+q}\left(\underline{\lim }_{\alpha} L_{\alpha}, M\right),
$$

as one can see by replacing $M$ with its right injective resolution and computing the derived functors of inductive and projective limits $\lim _{\alpha}^{p} L_{\alpha}$ and $\lim _{\alpha}^{p} \operatorname{Ext}_{A}^{q}\left(L_{\alpha}, M\right)$ in terms of the bar-constructions. In particular, if $\overrightarrow{\operatorname{Ext}}_{A}^{\alpha}\left(L_{\alpha}, M\right)=0$ for all $\alpha$ and all $q>0$, then $\operatorname{Ext}_{A}^{p}\left(\lim _{\alpha} L_{\alpha}, M\right) \simeq \lim _{\alpha}^{p} \operatorname{Hom}_{A}\left(L_{\alpha}, M\right)$. It remains to recall that the homological dimension of the derived functor of projective limit along a filtered poset of the cardinality $\aleph_{n}$ does not exceed $n+1[11]$. 
The following result is to be compared to the discussion of contraderived categories over coherent CDG-rings in [16, Section 3.8].

Theorem 2.4. Let $A$ be a left coherent ring such that any fp-injective left $A$-module has finite injective dimension. Then the triangulated functor $\operatorname{Hot}\left(A-\bmod _{\mathrm{inj}}\right) \longrightarrow$ $\mathrm{D}^{\mathrm{co}}(A-\mathrm{mod})$ induced by the embedding of additive/exact categories $A-$ mod $_{\mathrm{inj}} \longrightarrow$ $A-\bmod i s$ an equivalence of triangulated categories.

Proof. Moreover, for any CDG-ring $(A, d, h)$ whose underlying graded $\operatorname{ring} A$ is left coherent and has the property that the injective dimensions of fp-injective graded left modules over it are finite, the homotopy category of left CDG-modules over $(A, d, h)$ with injective underlying graded left $A$-modules is equivalent to the coderived category of CDG-modules. Indeed, according to [16, Section 3.7] it suffices that countable direct sums of injective (graded) $A$-modules be of finite injective dimensions, so it remains to recall that direct sums of injective modules are fp-injective.

Notice that the coderived category of $A$-modules in the sense of Becker [1, Proposition 1.3.6(2)] is defined as the homotopy category of complexes of injective $A$-modules (or the coderived category of CDG-modules over $A$ is defined as the homotopy category of CDG-modules with injective underlying graded $A$-modules, in the case of a CDG-ring $A=(A, d, h)$ ). Hence our Theorem 2.4, when its homological dimension condition is satisfied, makes the results of Št'ovíček [22, Section 6] about Becker's coderived category of complexes of modules over a coherent ring (or complexes of objects of a locally coherent Grothendieck category) applicable to the coderived category in our sense. In particular, our compact generation result in Corollary 2.6(b) below becomes a particular case of [22, Corollary 6.13].

Furthermore, it is instructive to compare the result of our Theorem 2.2 with that of [22, Theorem 6.12]. According to Theorem 2.2, our coderived category of the abelian category of left $A$-modules is equivalent to our coderived category of the exact category of fp-injective left $A$-modules. According to [22, Theorem 6.12], Becker's coderived category of the abelian category of left $A$-modules is equivalent to the conventional derived category of the exact category of fp-injective left $A$-modules. In both cases, it is only assumed that the ring $A$ is left coherent.

Lemma 2.5. Let $A$ be a left coherent ring. Then

(a) for any bounded complex $P^{\bullet}$ of finitely presented left A-modules and any complex $J^{\bullet}$ of fp-injective left A-modules, the Hom complex $\operatorname{Hom}_{A}\left(P^{\bullet}, J^{\bullet}\right)$ computes the groups $\operatorname{Hom}_{\mathrm{D}^{\mathrm{co}}(A-\bmod )}\left(P^{\bullet}, J^{\bullet}[*]\right)$;

(b) assuming that fp-injective left $A$-modules have finite injective dimensions, for any complex $P^{\bullet}$ of fp-projective left $A$-modules and any complex $J^{\bullet}$ of fp-injective left A-modules the complex $\operatorname{Hom}_{A}\left(P^{\bullet}, J^{\bullet}\right)$ computes the groups $\operatorname{Hom}_{\mathrm{D}^{\mathrm{co}}(A-\bmod )}\left(P^{\bullet}, J^{\bullet}[*]\right)$.

Proof. According to (the proof of) Theorem 2.2, any complex of left $A$-modules admits a morphism with a coacyclic cone into a complex of fp-injective left $A$-modules, and any complex of fp-injective left $A$-modules that is coacyclic with respect to the abelian category of arbitrary left $A$-modules is also coacyclic with respect to the 
exact category of fp-injective left $A$-modules. Hence in both parts (a) and (b) it suffices to prove that the complex $\operatorname{Hom}_{A}\left(P^{\bullet}, J^{\bullet}\right)$ is acyclic when (the complex $P^{\bullet}$ satisfies the respective condition and) $J^{\bullet}$ is a coacyclic complex of fp-injective left $A$-modules. Furthermore, in the assumption of (b) the exact category of fp-injective left $A$-modules has finite homological dimension, so any coacyclic (and even any acyclic) complex in it is absolutely acyclic [14, Remark 2.1]. It remains to notice that the complex $\operatorname{Hom}_{A}$ from a complex of fp-projective left $A$-modules to the total complex of a short exact sequence of complexes of fp-injective left $A$-modules is acyclic, and the functor $\mathrm{Hom}_{A}$ from a bounded complex of finitely generated left $A$-modules takes infinite direct sums of complexes of left $A$-modules to infinite direct sums of complexes of abelian groups.

Corollary 2.6. Let $A$ be a left coherent ring. Then

(a) the full subcategory of bounded complexes of finitely presented left A-modules $\mathrm{D}^{\mathrm{b}}\left(A-\bmod _{\mathrm{fp}}\right) \subset \mathrm{D}^{\mathrm{co}}(A$-mod $)$ in the coderived category of left $A$-modules consists of compact objects in $\mathrm{D}^{\mathrm{co}}(A-\bmod )$;

(b) assuming that $f p$-injective left $A$-modules have finite injective dimensions, the coderived category $\mathrm{D}^{\mathrm{co}}(A$-mod $)$ is compactly generated, and its full subcategory $\mathrm{D}^{\mathrm{b}}\left(A-\bmod _{\mathrm{fp}}\right)$ is precisely its full subcategory of compact objects.

Proof. By [18, Lemma A.1.2], the full subcategory of bounded below complexes in $\mathrm{D}^{\mathrm{co}}(A-\mathrm{mod})$ is equivalent to the category $\mathrm{D}^{+}(A-\mathrm{mod})$, in which $\mathrm{D}^{\mathrm{b}}\left(A-\bmod _{\mathrm{fp}}\right) \subset$ $\mathrm{D}^{\mathrm{b}}(A$-mod $) \subset \mathrm{D}^{+}(A$-mod $)$ is a full subcategory. Hence the full subcategory of bounded complexes of finitely presented left $A$-modules in $\mathrm{D}^{\mathrm{co}}(A$-mod $)$ is indeed equivalent to $\mathrm{D}^{\mathrm{b}}\left(A-\bmod _{\mathrm{fp}}\right)$. Since the class of fp-injective modules is closed under infinite direct sums in $A$-mod, the rest of part (a) follows from Lemma 2.5(a).

In part $(\mathrm{b})$, it is clear that the category $\mathrm{D}^{\mathrm{b}}\left(A-\bmod _{\mathrm{fp}}\right)$ contains the images of its idempotent endomorphisms, since so does the derived category $\mathrm{D}(A$-mod $)$, where $\mathrm{D}^{\mathrm{b}}\left(A-\bmod _{\mathrm{fp}}\right)$ is an idempotent closed subcategory by Lemma 1.2. So it remains to show that any complex $C^{\bullet}$ of left $A$-modules such that $\operatorname{Hom}_{\mathrm{D}^{\mathrm{co}}(A-\bmod )}\left(P^{\bullet}, C^{\bullet}\right)=0$ for all $P^{\bullet} \in \mathrm{D}^{\mathrm{b}}\left(A-\bmod _{\mathrm{fp}}\right)$ vanishes in $\mathrm{D}^{\mathrm{co}}(A-\bmod )$. Here one can argue as in $[7$, Lemma 2.2]. Represent the object $C^{\bullet} \in \mathrm{D}^{\mathrm{co}}(A$-mod) by a complex of fp-injective left $A$-modules $J^{\bullet}$; then $\operatorname{Hom}_{\mathrm{D}^{\text {co }}(A \text {-mod })}\left(P^{\bullet}, C^{\bullet}[*]\right)$ can be computed as $\operatorname{Hom}_{A}\left(P^{\bullet}, J^{\bullet}\right)$.

If the complex $J^{\bullet}$ (or $C^{\bullet}$ ) has a nonzero cohomology module $H^{n} J^{\bullet} \neq 0$ in some degree $n$, then there exists a finitely presented $A$-module $P$ (e. g., $P=A$ ) and a morphism of complexes $P \longrightarrow J^{\bullet}[n]$ inducing a nonzero map of the cohomology modules. Otherwise, when $H^{*}\left(J^{\bullet}\right)=0$, one has $H^{n+1} \operatorname{Hom}_{A}\left(P, J^{\bullet}\right) \simeq \operatorname{Ext}_{A}^{1}\left(P, Z^{n}\right)$, where $Z^{n}$ is the kernel of the differential $J^{n} \longrightarrow J^{n+1}$, for any finitely presented left $A$-module $P$. If $\operatorname{Ext}_{A}^{1}\left(P, Z^{n}\right)=0$ for all $P$ and $n$, then the $A$-modules $Z^{n}$ are fp-injective and the complex $J^{\bullet}$ is acyclic in the exact category $A-\bmod _{\text {fpi }}$. Since by the assumption of (b) this exact category has finite homological dimension, by [14, Remark 2.1] the complex $J^{\bullet}$ is coacyclic (and even absolutely acyclic) in $A-\bmod _{\mathrm{fpi}}$. (Cf. [22, Proposition 6.4], where a similar argument is presented without any homological dimension assumptions.) 


\section{Dualizing Complexes and Contravariant Duality}

The notion of a bimodule over an arbitrary pair of rings is inherently problematic from the homological point of view. It suffices to consider the example of $A$-B-bimodules for the pair of rings $A=\mathbb{Z} / 4$ and $B=\mathbb{Z} / 2$ in order to see where the problem lies. In particular, it is not always possible to embed an $A$ - $B$-module into an $A$-injective $A$ - $B$-bimodule. Restricting to the case $A=B$ does not help much, as the trouble repeats itself for the $\operatorname{ring} A=B=\mathbb{Z} / 2 \oplus \mathbb{Z} / 4$. Assuming that both $A$ and $B$ are flat algebras over the same commutative ring $k$ and working with $A$ - $B$-bimodules over $k$ (i. e., left modules over $A \otimes_{k} B^{\text {op }}$ ) resolves the problem.

One has to deal with this issue when defining the notion of a dualizing complex over a pair of noncommutative rings. Several approaches have been tried in the literature, from restricting outright to the case of algebras over a field [25, 26] to specifying explicit left and right adjustness conditions on complexes of bimodules [2, 12, 18]. In this section we show that the most naïve weak definition of a dualizing complex works well enough to provide a contravariant equivalence between bounded derived categories of finitely presented modules.

Given two associative rings $A$ and $B$, denote by $A$-mod- $B$ the abelian category of $A$-B-bimodules. Let $A$ be a left coherent ring and $B$ be a right coherent ring. A complex of $A$-B-bimodules $D^{\bullet} \in \mathrm{D}(A$-mod-B $)$ is said to be a weak dualizing complex for $A$ and $B$ if the following conditions are satisfied:

$\left(\mathrm{i}^{\mathrm{w}}\right)$ as a complex of left $A$-modules, $D^{\bullet}$ is quasi-isomorphic to a finite complex of injective $A$-modules; and as a complex of right $B$-modules, $D^{\bullet}$ is quasiisomorphic to a finite complex of injective $B$-modules;

(ii) the $A$-B-bimodules of cohomology $H^{*}\left(D^{\bullet}\right)$ of the complex $D^{\bullet}$ are finitely presented left $A$-modules and finitely presented right $B$-modules;

(iii) the homothety maps $A \longrightarrow \operatorname{Hom}_{\mathrm{D}(\bmod -B)}\left(D^{\bullet}, D^{\bullet}[*]\right)$ and $B^{\mathrm{op}} \longrightarrow \operatorname{Hom}_{\mathrm{D}(A-\bmod )}$ $\left(D^{\bullet}, D^{\bullet}[*]\right)$ are isomorphisms of graded rings.

The following result is a slight generalization of [25, Propositions 3.4-3.5] and [12, first assertion of Corollary 2.8].

Theorem 3.1. Let $D^{\bullet}$ be a weak dualizing complex for a left coherent ring $A$ and a right coherent ring $B$. Then there is an anti-equivalence between the bounded derived categories $\mathrm{D}^{\mathrm{b}}\left(A-\bmod _{\mathrm{fp}}\right)$ and $\mathrm{D}^{\mathrm{b}}\left(\bmod _{\mathrm{fp}}-B\right)$ of finitely presented left $A$-modules and finitely presented right $B$-modules provided by the mutually inverse functors $\mathbb{R} \operatorname{Hom}_{A}\left(-, D^{\bullet}\right)$ and $\mathbb{R} \operatorname{Hom}_{B^{\mathrm{op}}}\left(-, D^{\bullet}\right)$.

Proof. Let $A-$ mod $_{\text {proj }} \subset A$-mod denote the additive category of projective left $A$-modules and let $\operatorname{Hot}^{-}\left(A-\bmod _{\text {proj }}\right)$ be its bounded above homotopy category. Then the bounded derived category of left $A$-modules $\mathrm{D}^{\mathrm{b}}(A$-mod) can be identified with the full subcategory in $\operatorname{Hot}^{-}\left(A-\bmod _{\text {proj }}\right)$ consisting of complexes with bounded cohomology. Restricting the contravariant functor

$$
\operatorname{Hom}_{A}\left(-, D^{\bullet}\right): \operatorname{Hot}(A-\bmod )^{\text {op }} \longrightarrow \operatorname{Hot}(\bmod -B)
$$


acting between the homotopy categories of left $A$-modules and right $B$-modules to the full subcategory

$$
\mathrm{D}^{\mathrm{b}}(A-\mathrm{mod}) \subset \operatorname{Hot}^{-}\left(A-\bmod _{\text {proj }}\right) \subset \operatorname{Hot}(A-\bmod ),
$$

we obtain the derived functor $\mathbb{R} \operatorname{Hom}_{A}\left(-, D^{\bullet}\right): \mathrm{D}^{\mathrm{b}}(A-\bmod )^{\mathrm{op}} \longrightarrow \mathrm{D}(\bmod -B)$.

Let us show that the image of this functor is contained in the bounded derived category $\mathrm{D}^{\mathrm{b}}(\bmod -B) \subset \mathrm{D}(\bmod -B)$ of right $B$-modules. Indeed, the property of a complex of $B$-modules to have bounded cohomology only depends on its underlying complex of abelian groups. The composition $\mathrm{D}^{\mathrm{b}}(A-\bmod )^{\mathrm{op}} \longrightarrow \mathrm{D}(\bmod -B) \longrightarrow \mathrm{D}(\mathrm{Ab})$ of the functor $\mathbb{R} \operatorname{Hom}_{A}$ with the forgetful functor $\mathrm{D}(\bmod -B) \longrightarrow \mathrm{D}(\mathrm{Ab})$ to the derived category of abelian groups can be computed as the functor $\operatorname{Hom}_{A}\left(-{ }^{\prime} D^{\bullet}\right)$, where ${ }^{\prime} D^{\bullet}$ is a finite complex of injective left $A$-modules quasi-isomorphic to $D^{\bullet}$. The latter functor obviously takes $\mathrm{D}^{\mathrm{b}}(A$-mod $)$ to $\mathrm{D}^{\mathrm{b}}(\mathrm{Ab})$.

We have obtained the right derived functor $\mathbb{R} \operatorname{Hom}_{A}\left(-, D^{\bullet}\right): \mathrm{D}^{\mathrm{b}}(A-\bmod )^{\text {op }} \longrightarrow$ $\mathrm{D}^{\mathrm{b}}(\bmod -B)$; similarly, there is the right derived functor $\mathbb{R} \operatorname{Hom}_{B^{\text {op }}}\left(-, D^{\bullet}\right)$ : $\mathrm{D}^{\mathrm{b}}(\bmod -B)^{\mathrm{op}} \longrightarrow \mathrm{D}^{\mathrm{b}}(A-\bmod )$. Let us show that these two contravariant functors are right adjoint to each other; in other words, for any complexes $M^{\bullet} \in \mathrm{D}^{\mathrm{b}}(A$-mod) and $N^{\bullet} \in \mathrm{D}^{\mathrm{b}}(\bmod -B)$ there is a natural isomorphism of abelian groups

$$
\operatorname{Hom}_{\mathrm{D}^{\mathrm{b}(\bmod -B)}}\left(N^{\bullet}, \mathbb{R} \operatorname{Hom}_{A}\left(M^{\bullet}, D^{\bullet}\right)\right) \simeq \operatorname{Hom}_{\mathrm{D}^{\mathrm{b}}(A-\bmod )}\left(M^{\bullet}, \mathbb{R} \operatorname{Hom}_{B^{\text {op }}}\left(N^{\bullet}, D^{\bullet}\right)\right) .
$$

Indeed, represent the object $M^{\bullet}$ by a bounded above complex of projective left $A$-modules $P^{\bullet}$ and the object $N^{\bullet}$ by a bounded above complex of projective right $B$-modules $Q^{\bullet}$; then the passage to the degree-zero cohomology groups in the natural isomorphism of complexes $\operatorname{Hom}_{B^{\text {op }}}\left(Q^{\bullet}, \operatorname{Hom}_{A}\left(P^{\bullet}, D^{\bullet}\right)\right) \simeq$ $\operatorname{Hom}_{A}\left(P^{\bullet}, \operatorname{Hom}_{B^{\text {op }}}\left(Q^{\bullet}, D^{\bullet}\right)\right)$ provides the desired isomorphism of the Hom groups in the derived categories.

Furthermore, let us check that the functor $\operatorname{Hom}_{A}\left(-, D^{\bullet}\right)$ takes the bounded derived category of finitely presented left $A$-modules $\mathrm{D}^{\mathrm{b}}\left(A-\bmod _{\mathrm{fp}}\right) \subset \mathrm{D}^{\mathrm{b}}(A$-mod $)$ into the bounded derived category of finitely presented right $B$-modules $\mathrm{D}^{\mathrm{b}}\left(\bmod _{\mathrm{fp}}-B\right) \subset$ $\mathrm{D}^{\mathrm{b}}(\bmod -B)$ (see Corollary 1.3$)$. Indeed, an object of $\mathrm{D}^{\mathrm{b}}\left(A-\bmod _{\mathrm{fp}}\right)$ can be represented by a bounded above complex of finitely generated projective left $A$-modules $P^{\bullet}$, and the complex of $A$-B-bimodules with $D^{\bullet}$ with bounded cohomology can be replaced by a quasi-isomorphic finite complex of $A$ - $B$-bimodules. Then the property of every cohomology module of the complex $\operatorname{Hom}_{A}\left(P^{\bullet}, D^{\bullet}\right)$ to be finitely presented over $B$ only depends on a finite fragment of the complex $P^{\bullet}$, which reduces the question to the case of a one-term complex $P^{\bullet}=P$ corresponding to a finitely generated projective $A$-module $P$. It remains to recall that the cohomology bimodules of the complex $D^{\bullet}$ were assumed to be finitely presented right $B$-modules.

We have constructed the derived functor

$$
\mathbb{R} \operatorname{Hom}_{A}\left(-, D^{\bullet}\right): \mathrm{D}^{\mathrm{b}}\left(A-\bmod _{\mathrm{fp}}\right)^{\mathrm{op}} \longrightarrow \mathrm{D}^{\mathrm{b}}\left(\bmod _{\mathrm{fp}}-B\right) ;
$$

similarly one obtains the derived functor

$$
\mathbb{R} \operatorname{Hom}_{B^{\circ \mathrm{p}}}\left(-, D^{\bullet}\right): \mathrm{D}^{\mathrm{b}}\left(\bmod _{\mathrm{fp}}-B\right)^{\mathrm{op}} \longrightarrow \mathrm{D}^{\mathrm{b}}\left(A-\bmod _{\mathrm{fp}}\right) .
$$


It remains to prove that these are mutually inverse anti-equivalences. For this purpose, we will show that the adjunction maps are quasi-isomorphisms; it suffices to check that these are quasi-isomorphisms of complexes of abelian groups.

Let an object of the derived category $\mathrm{D}^{\mathrm{b}}\left(A-\bmod _{\mathrm{fp}}\right)$ be represented by a bounded above complex of finitely generated projective left $A$-modules $P^{\bullet}$. Replace the complex $D^{\bullet}$ by a quasi-isomorphic finite complex of $A$ - $B$-bimodules; and let " $D$ ' be a finite complex of injective right $B$-modules endowed with a quasi-isomorphism of complexes of right $B$-modules $D^{\bullet} \longrightarrow{ }^{\prime \prime} D^{\bullet}$. Then checking that the natural map of complexes of abelian groups $P^{\bullet} \longrightarrow \operatorname{Hom}_{B^{\text {op }}}\left(\operatorname{Hom}_{A}\left(P^{\bullet}, D^{\bullet}\right),{ }^{\prime \prime} D^{\bullet}\right)$ is a quasi-isomorphism reduces to the case of a one-term complex $P^{\bullet}=A$, when the desired assertion becomes an expression of the assumption that the homothety map $A \longrightarrow \operatorname{Hom}_{\mathrm{D}(\bmod -B)}\left(D^{\bullet}, D^{\bullet}[*]\right)$ is an isomorphism of graded rings.

Notice that the construction of the duality functors between the categories $\mathrm{D}^{\mathrm{b}}\left(A-\bmod _{\mathrm{fp}}\right)$ and $\mathrm{D}^{\mathrm{b}}\left(\bmod _{\mathrm{fp}}-B\right)$ becomes much simpler when $D^{\bullet}$ is a finite complex of fp-injective left $A$-modules and right $B$-modules (cf. the definition of a dualizing complex in the next Section 4). In this case one can simply apply the underived functors $\operatorname{Hom}_{A}\left(-, D^{\bullet}\right)$ and $\operatorname{Hom}_{B^{\text {op }}}\left(-, D^{\bullet}\right)$ to bounded complexes of finitely presented left $A$-modules and right $B$-modules, obtaining complexes of right $B$-modules and left $A$-modules with bounded and finitely presented cohomology modules (which form categories equivalent to $\mathrm{D}^{\mathrm{b}}\left(\bmod _{\mathrm{fp}}-B\right)$ and $\mathrm{D}^{\mathrm{b}}\left(A-\bmod _{\mathrm{fp}}\right)$ by Corollary 1.3$)$.

The following definition can be found in [12] (see also [18, Section B.4]). Let $A$ be a left coherent ring and $B$ be a right coherent ring. A finite complex of $A$ - $B$-bimodules $D^{\bullet}$ is said to be a strong dualizing complex for the rings $A$ and $B$ if the following conditions are satisfied:

(is ) the terms of the complex $D^{\bullet}$ are injective left $A$-modules and injective right $B$-modules;

(ii) the $A$-B-bimodules of cohomology $H^{*}\left(D^{\bullet}\right)$ of the complex $D^{\bullet}$ are finitely presented left $A$-modules and finitely presented right $B$-modules;

(iii $\left.{ }^{\mathrm{s}}\right)$ the homothety maps $A \longrightarrow \operatorname{Hom}_{B^{\text {op }}}\left(D^{\bullet}, D^{\bullet}\right)$ and $B^{\text {op }} \longrightarrow \operatorname{Hom}_{A}\left(D^{\bullet}, D^{\bullet}\right)$ are quasi-isomorphisms of DG-rings.

The condition (ii) is the same as in the above definition of a weak dualizing complex. In the assumption of the condition $\left(\mathrm{i}^{\mathrm{s}}\right)$, the condition $\left(\mathrm{iii}^{\mathrm{s}}\right)$ is an equivalent restatement of the condition (iii). The following result is the second assertion of [12, Corollary 2.8]; see also [26, Proposition 1.3].

Theorem 3.2. Let $D^{\bullet}$ be a strong dualizing complex for a left coherent ring $A$ and a right coherent ring $B$. Then there is an anti-equivalence between the derived categories of unbounded complexes of left $A$-modules and right $B$-modules with finitely presented cohomology modules $\mathrm{D}_{\mathrm{fp}}(A-\bmod )$ and $\mathrm{D}_{\mathrm{fp}}(\bmod -B)$ provided by the mutually inverse functors $\operatorname{Hom}_{A}\left(-, D^{\bullet}\right)$ and $\operatorname{Hom}_{B^{\circ \mathrm{p}}}\left(-, D^{\bullet}\right)$.

Proof. In the assumption $\left(\mathrm{i}^{\mathrm{s}}\right)$, the functors $\operatorname{Hom}_{A}\left(-, D^{\bullet}\right): \operatorname{Hot}(A-\bmod )^{\text {op }} \longrightarrow$ $\operatorname{Hot}(\bmod -B)$ and $\operatorname{Hom}_{B^{\text {op }}}\left(-, D^{\bullet}\right): \operatorname{Hot}(\bmod -B)^{\text {op }} \longrightarrow \operatorname{Hot}(A-\bmod )$ take acyclic 
complexes to acyclic complexes and consequently induce triangulated functors

$$
\operatorname{Hom}_{A}\left(-, D^{\bullet}\right): \mathrm{D}(A-\bmod )^{\text {op }} \longrightarrow \mathrm{D}(\bmod -B)
$$

and

$$
\operatorname{Hom}_{B^{\mathrm{op}}}\left(-, D^{\bullet}\right): \mathrm{D}(\bmod -B)^{\mathrm{op}} \longrightarrow \mathrm{D}(A-\bmod ) .
$$

It is not difficult to see that these two contravariant functors are right adjoint to each other. Now verifying that these functors take the full subcategories $\mathrm{D}_{\mathrm{fp}}(A$-mod $) \subset$ $\mathrm{D}(A-\bmod )$ and $\mathrm{D}_{\mathrm{fp}}(\bmod -B) \subset \mathrm{D}(\bmod -B)$ into each other and the adjunction morphisms are quasi-isomorphisms for complexes from these subcategories depends only on finite fragments of the complexes involved, which makes these questions straightforward (and certainly easier than the ones resolved in the previous proof).

Example 3.3. Let $K \longrightarrow A$ be an associative ring homomorphism such that $A$ is a finitely generated projective left $K$-module. Set $\mathcal{C}=\operatorname{Hom}_{K}(A, K)$; then the map $\mathrm{C} \longrightarrow \mathrm{C} \otimes_{K} \mathrm{C}$ dual to the multiplication map $A \otimes_{K} A \longrightarrow A$ endows the $K$ - $K$-bimodule $\mathrm{C}$ with the structure of a coassociative coring over $K$. The $K$ - $K$-bimodule $\mathcal{C}$ is a finitely generated projective right $K$-module by construction; suppose further that it is also a finitely generated projective left $K$-module. Set $B=\operatorname{Hom}_{K}(\mathcal{C}, K)$; then the map $B \otimes_{K} B \longrightarrow B$ dual to the comultiplication map $\mathcal{C} \longrightarrow \mathcal{C} \otimes_{K} \mathcal{C}$ endows the $K$-K-bimodule $B$ with the structure of an associative ring. The unit map/ring homomorphism $K \longrightarrow A$ is transformed into a counit map $\mathrm{C} \longrightarrow K$ and into a unit map/ring homomorphism $K \longrightarrow B$.

The category of left $A$-modules is isomorphic to the category of left $\mathcal{C}$-comodules, and the category of right $B$-modules is isomorphic to the category of right C-comodules. So, in particular, the natural $\mathcal{C}$-C-bicomodule structure on $\mathcal{C}$ can be viewed as an $A$ - $B$-bimodule structure (see [14, Section 10.1.1]; cf. [15, Section 3.2]).

Assume that the ring $K$ is left and right coherent, and suppose further that $K$ is a left and right Gorenstein ring, i. e., $K$ has a finite injective dimension as a left and right module over itself. Then the $K-K$-bimodule $K$ is a weak dualizing complex for the rings $K$ and $K$ [26, Example 2.3(a)], while the $A$-B-bimodule $\mathcal{C}$ is a weak dualizing complex for the rings $A$ and $B$ (cf. [25, Section 5] and [16, Section 3.10]). When the ring $K$ is classically semisimple, the $A$ - $B$-bimodule $\mathrm{C}$ is even a strong dualizing complex for the rings $A$ and $B$.

Example 3.4. Let $K$ be a commutative ring and $A$ be an associative $K$-algebra (with unit). Assume that the ring $K$ is coherent and the $\operatorname{ring} A$ is a finitely generated projective $K$-module. Let $D_{K}^{\bullet}$ be a (weak or strong) dualizing complex for the ring $K$, i. e., a complex of $K$-modules that, viewed as a complex of $K$ - $K$-bimodules, is a (weak or strong) dualizing complex for the rings $K$ and $K$. Then the complex of $A$-A-bimodules $\operatorname{Hom}_{K}\left(A, D_{K}^{\bullet}\right)$ is a (respectively, weak or strong) dualizing complex for the rings $A$ and $A$ (cf. [25, Example 3.8 and Corollary 5.6]). 


\section{Covariant Duality Theorem}

The aim of this section is to extend the noncommutative covariant SerreGrothendieck duality theory developed in the papers $[6,7,8]$ from Noetherian to coherent rings. Here is our main definition in this context.

Let $A$ be a left coherent ring and $B$ be a right coherent ring. A finite complex of $A$-B-bimodules $D^{\bullet}$ is called a dualizing complex for the rings $A$ and $B$ if it satisfies the following conditions:

(i) the terms of the complex $D^{\bullet}$ are fp-injective left $A$-modules and fp-injective right $B$-modules;

(ii) the $A$-B-bimodules of cohomology $H^{*}\left(D^{\bullet}\right)$ of the complex $D^{\bullet}$ are finitely presented left $A$-modules and finitely presented right $B$-modules;

(iii) the homothety maps $A \longrightarrow \operatorname{Hom}_{\mathrm{D}(\bmod -B)}\left(D^{\bullet}, D^{\bullet}[*]\right)$ and $B^{\text {op }} \longrightarrow \operatorname{Hom}_{\mathrm{D}(A-\bmod )}$ $\left(D^{\bullet}, D^{\bullet}[*]\right)$ are isomorphisms of graded rings.

The conditions (ii-iii) are the same as in the definition of a weak dualizing complex in Section 3. The following lemma explains how the fp-injectivity condition in (i) is to be used. For the rest of this section, we assume that the ring $A$ is left coherent and the ring $B$ is right coherent.

Lemma 4.1. (a) Let $F$ be a flat left $B$-module and $E$ be an A-fp-injective $A$-B-bimodule. Then the tensor product $E \otimes_{B} F$ is an fp-injective left $A$-module.

(b) Let $J$ be an injective left $A$-module and $E$ be a $B$-fp-injective $A$-B-bimodule. Then the left $B$-module $\operatorname{Hom}_{A}(E, J)$ is flat.

Proof. In part (a), one can check that the natural map $\operatorname{Hom}_{A}(M, E) \otimes_{B} F \longrightarrow$ $\operatorname{Hom}_{A}\left(M, E \otimes_{B} F\right)$ is an isomorphism for any finitely presented left $A$-module $M$. (Alternatively, one can use the Govorov-Lazard characterization of flat modules as filtered inductive limits of finitely generated projective ones together with the fact that the class of fp-injective left modules over a left coherent ring is closed under filtered inductive limits.) In part (b), one needs to show that the natural map $N \otimes_{B} \operatorname{Hom}_{A}(E, J) \longrightarrow \operatorname{Hom}_{A}\left(\operatorname{Hom}_{B^{\mathrm{op}}}(N, E), J\right)$ is an isomorphism for any finitely presented right $B$-module $N$.

The next lemma is a generalization of [18, Lemma B.4.1]. We assume that $D^{\bullet}$ is a dualizing complex for the rings $A$ and $B$.

Lemma 4.2. (a) Let $F$ be a flat left B-module and $J^{\bullet}$ be a bounded below complex of injective left A-modules endowed with a quasi-isomorphism of complexes of left A-modules $D^{\bullet} \otimes_{B} F \longrightarrow J^{\bullet}$. Then the natural morphism of complexes of left $B$-modules $F \longrightarrow \operatorname{Hom}_{A}\left(D^{\bullet}, J^{\bullet}\right)$ is a quasi-isomorphism.

(b) Let $J$ be an injective left A-module. Then the natural morphism of complexes of left A-modules $D^{\bullet} \otimes_{B} \operatorname{Hom}_{A}\left(D^{\bullet}, J\right) \longrightarrow J$ is a quasi-isomorphism.

Proof. Part (a): let ' $D$ • be a bounded above complex of finitely generated projective left $A$-modules endowed with a quasi-isomophism of complexes of left $A$-modules ${ }^{\prime} D^{\bullet} \longrightarrow D^{\bullet}$. Then the natural morphism $\operatorname{Hom}_{A}\left(D^{\bullet}, J^{\bullet}\right) \longrightarrow \operatorname{Hom}_{A}\left({ }^{\prime} D^{\bullet}, J^{\bullet}\right)$ 
is a quasi-isomorphism of complexes of abelian groups, as is the natural morphism $\operatorname{Hom}_{A}\left({ }^{\prime} D^{\bullet}, D^{\bullet} \otimes_{B} F\right) \longrightarrow \operatorname{Hom}_{A}\left({ }^{\prime} D^{\bullet}, J^{\bullet}\right)$. The square of morphisms of complexes of abelian groups $F \longrightarrow \operatorname{Hom}_{A}\left(D^{\bullet}, J^{\bullet}\right) \longrightarrow \operatorname{Hom}_{A}\left({ }^{\prime} D^{\bullet}, J^{\bullet}\right)$ and $F \longrightarrow \operatorname{Hom}_{A}\left({ }^{\prime} D^{\bullet}, D^{\bullet} \otimes_{B} F\right) \longrightarrow \operatorname{Hom}_{A}\left({ }^{\prime} D^{\bullet}, J^{\bullet}\right)$ is commutative, so it suffices to show that the morphism $F \longrightarrow \operatorname{Hom}_{A}\left({ }^{\prime} D^{\bullet}, D^{\bullet} \otimes_{B} F\right)$ is a quasi-isomorphism. Now the complex of abelian groups $\operatorname{Hom}_{A}\left({ }^{\prime} D^{\bullet}, D^{\bullet} \otimes_{B} F\right)$ is isomorphic to $\operatorname{Hom}_{A}\left({ }^{\prime} D^{\bullet}, D^{\bullet}\right) \otimes_{B} F$, and it remains to recall that the map $B \longrightarrow \operatorname{Hom}_{A}\left({ }^{\prime} D^{\bullet}, D^{\bullet}\right)$ induced by the right action of $B$ in $D^{\bullet}$ is a quasi-isomorphism of complexes of right $B$-modules by the assumption (iii).

Part (b): let " $D$ • be a bounded above complex of finitely generated projective right $B$-modules endowed with a quasi-isomophism of complexes of right $B$-modules $" D^{\bullet} \longrightarrow D^{\bullet}$. Then the natural morphism " $D^{\bullet} \otimes_{B} \operatorname{Hom}_{A}\left(D^{\bullet}, J\right) \longrightarrow D^{\bullet} \otimes_{B}$ $\operatorname{Hom}_{A}\left(D^{\bullet}, J\right)$ is a quasi-isomorphism of complexes of abelian groups, and it suffices to show that the composition " $D^{\bullet} \otimes_{B} \operatorname{Hom}_{A}\left(D^{\bullet}, J\right) \longrightarrow D^{\bullet} \otimes_{B} \operatorname{Hom}_{A}\left(D^{\bullet}, J\right) \longrightarrow J$ is also a quasi-isomorphism of complexes of abelian groups. Now the complex of abelian groups " $D^{\bullet} \otimes_{B} \operatorname{Hom}_{A}\left(D^{\bullet}, J\right)$ is isomorphic to $\operatorname{Hom}_{A}\left(\operatorname{Hom}_{B^{\text {op }}}\left({ }^{\prime \prime} D^{\bullet}, D^{\bullet}\right), J\right)$, and it remains to recall that the map $A \longrightarrow \operatorname{Hom}_{B^{\text {op }}}\left({ }^{\prime \prime} D^{\bullet}, D^{\bullet}\right)$ is a quasi-isomorphism of complexes of left $A$-modules by the assumption (iii).

The following result generalizes the first assertion of [2, Proposition 1.5] (see also [18, Corollary B.4.2]). It can be used in conjunction with Proposition 2.3.

Proposition 4.3. Let $D^{\bullet}$ be a dualizing complex for a left coherent ring $A$ and a right coherent ring $B$. Then the supremum of projective dimensions of flat left $B$-modules cannot exceed the supremum of injective dimensions of fp-injective left A-modules by more than the length of the complex $D^{\bullet}$.

Proof. Assume that the complex $D^{\bullet}$ is concentrated in the cohomological degrees from $i$ to $i+d$, and the supremum of injective dimensions of fp-injective left $A$-modules is a finite integer $n$. In order to show that the projective dimension of any flat left $B$-module does not exceed $n+d$, it suffices to check that $\operatorname{Ext}_{B}^{n+d+1}(F, G)=0$ for any flat left $B$-modules $F$ and $G$. Let $P$. be a left projective resolution of the $B$-module $F$ and $J^{\bullet}$ be a complex of injective left $A$-modules concentrated in the cohomological degrees from $i$ to $i+n+d$ and endowed with a quasi-isomorphism of complexes of left $A$-modules $D^{\bullet} \otimes_{B} G \longrightarrow J^{\bullet}$. By Lemma 4.2(a), the natural map of complexes of abelian groups

$$
\operatorname{Hom}_{B}\left(P_{\bullet}, G\right) \longrightarrow \operatorname{Hom}_{B}\left(P_{\bullet}, \operatorname{Hom}_{A}\left(D^{\bullet}, J^{\bullet}\right)\right)
$$

is a quasi-isomorphism. The right-hand side is isomorphic to the complex $\operatorname{Hom}_{A}\left(D^{\bullet} \otimes_{B} P_{\bullet}, J^{\bullet}\right)$, which is quasi-isomorphic to $\operatorname{Hom}_{A}\left(D^{\bullet} \otimes_{B} F, J^{\bullet}\right)$. The latter complex is concentrated in the degrees from $-d$ to $n+d$.

Denote by $B-\bmod _{\mathrm{fl}}$ the exact category of flat left $B$-modules and by $B-\bmod _{\text {proj }}$ the additive category of projective left $B$-modules. The following results, resembling Theorems 2.2 and 2.4, were established in our previous papers. 
Theorem 4.4. Let $B$ be a right coherent ring. Then

(a) the triangulated functor between the contraderived categories $\mathrm{D}^{\mathrm{ctr}}\left(B-\bmod _{\mathrm{fl}}\right) \longrightarrow$ $\mathrm{D}^{\mathrm{ctr}}(B$-mod $)$ induced by the embedding of exact categories $B-\bmod _{\mathrm{fl}} \longrightarrow B$-mod is an equivalence of triangulated categories;

(b) assuming that flat left $B$-modules have finite projective dimensions, the triangulated functor $\operatorname{Hot}\left(B-\bmod _{\text {proj }}\right) \longrightarrow \mathrm{D}^{\mathrm{ctr}}(B-\bmod )$ induced by the embedding of additive/exact categories $B$ - $\bmod _{\mathrm{proj}} \longrightarrow B$-mod is an equivalence of triangulated categories.

Proof. Notice that the class of flat left modules over a right coherent ring is closed under infinite products, so the triangulated functor in (a) is well-defined. The assertion of part (a) is provided by [17, Remark 1.5] and/or [18, Proposition A.3.1(b)]. Part (b) was proven in [16, Section 3.8]. In both cases, the same assertions also hold for the contraderived category of left CDG-modules over a CDG-ring $(B, d, h)$ with a right graded coherent underlying graded ring $B$, the contraderived category of CDG-modules with flat underlying graded modules, and the homotopy category of CDG-modules with projective underlying graded modules.

The next theorem, generalizing [8, Theorem 4.8] (see also [18, Corollary B.4.10 and Theorem D.2.5]), is one of the most important results of this paper.

Theorem 4.5. Let $D^{\bullet}$ be a dualizing complex for a left coherent ring $A$ and a right coherent ring $B$. Assume that fp-injective left A-modules have finite injective dimensions. Then there is an equivalence between the coderived category of left A-modules $\mathrm{D}^{\mathrm{co}}(A$-mod $)$ and the contraderived category of left $B$-modules $\mathrm{D}^{\mathrm{ctr}}(B$-mod $)$ provided by the mutually inverse functors $\mathbb{R} \operatorname{Hom}_{A}\left(D^{\bullet},-\right)$ and $D^{\bullet} \otimes_{B}^{\mathbb{L}}-$.

Proof. The derived functor $\mathbb{R} \operatorname{Hom}_{A}\left(D^{\bullet},-\right): \mathrm{D}^{\mathrm{co}}(A-\mathrm{mod}) \longrightarrow \mathrm{D}^{\mathrm{ctr}}(B-$ mod $)$ is constructed by identifying the coderived category $\mathrm{D}^{\mathrm{co}}(A-\bmod )$ with the homotopy category $\operatorname{Hot}\left(A-\bmod _{\text {inj }}\right)$ and applying the functor $\operatorname{Hom}_{A}\left(D^{\bullet},-\right)$ to complexes of injective left $A$-modules. By Lemma 4.1(b), this produces complexes of flat left $B$-modules.

The derived functor $D^{\bullet} \otimes_{B}^{\mathbb{L}}-: \mathrm{D}^{\mathrm{ctr}}(B-\mathrm{mod}) \longrightarrow \mathrm{D}^{\mathrm{co}}(A-\bmod )$ is obtained by identifying the contraderived category $\mathrm{D}^{\mathrm{ctr}}(B$-mod) of arbitrary left $B$-modules with the contraderived category $\mathrm{D}^{\mathrm{ctr}}\left(B-\bmod _{\mathrm{fl}}\right)$ of flat left $B$-modules and applying the functor $D^{\bullet} \otimes_{B}-$ to complexes of flat left $B$-modules. By Lemma 4.1(a), this produces complexes of fp-injective left $A$-modules. Since the category of flat left $B$-modules has finite homological dimension by the assumption of Theorem in view of Proposition 4.3, any contraacyclic complex of flat left $B$-modules is absolutely acyclic with respect to the exact category of flat left $B$-modules, and the functor $D^{\bullet} \otimes_{B}-$ transforms such a complex into an absolutely acyclic complex of fp-injective left $A$-modules; so the derived functor $D^{\bullet} \otimes_{B}^{\mathbb{L}}$ - is well-defined.

It is clear that the derived functor $D^{\bullet} \otimes_{B}^{\mathbb{L}}-$ is left adjoint to the derived functor $\mathbb{R} \operatorname{Hom}_{A}\left(D^{\bullet},-\right)$, so it remains to show that the adjunction morphisms are isomorphisms in $\mathrm{D}^{\mathrm{co}}\left(A\right.$-mod) and $\mathrm{D}^{\mathrm{ctr}}(B$-mod $)$. Let $J^{\bullet}$ be a complex of injective left $A$-modules. Then by Lemma $4.2(\mathrm{~b})$ the cone of the morphism of complexes of fp-injective left $A$-modules $D^{\bullet} \otimes_{B} \operatorname{Hom}_{A}\left(D^{\bullet}, J^{\bullet}\right) \longrightarrow J^{\bullet}$ is the total complex of a 
finite acyclic complex of complexes of fp-injective left $A$-modules, hence a coacyclic (and even an absolutely acyclic) complex of fp-injective left $A$-modules.

Let $F^{\bullet}$ be a complex of flat left $B$-modules. Consider the bicomplex of fp-injective left $A$-modules $D^{j} \otimes_{B} F^{i}$ and pick a bicomplex of injective left $A$-modules $J^{i j}$ together with a morphism of bicomplexes of $A$-modules $D^{\bullet} \otimes_{B} F^{\bullet} \longrightarrow J^{\bullet \bullet}$ such that the bicomplex $J^{\bullet \bullet}$ is concentrated in a finite interval of gradings $j$ and for every index $i$ the morphism of complexes $D^{\bullet} \otimes_{B} F^{i} \longrightarrow J^{i, \bullet}$ is a quasi-isomorphism. To obtain such a bicomplex $J^{\bullet \bullet}$ one can, e. g., use a functorial injective resolution construction in the category of left $A$-modules, or a construction of embedding of an arbitrary bicomplex in an abelian category with enough injectives into a bicomplex of injective objects. Denote simply by $\operatorname{Hom}_{A}\left(D^{\bullet}, J^{\bullet \bullet}\right)$ the totalization of the tricomplex $\operatorname{Hom}_{A}\left(D^{k}, J^{i j}\right)$ along the pair of indices $(j, k)$; then, by Lemma $4.2(\mathrm{a})$, the natural morphism of bicomplexes of flat left $B$-modules $F^{\bullet} \longrightarrow \operatorname{Hom}_{A}\left(D^{\bullet}, J^{\bullet \bullet}\right)$ is a quasi-isomorphism of finite (and uniformly bounded) complexes at every fixed degree $i$. Now the cone of the natural morphism between the totalizations of the bicomplexes $D^{\bullet} \otimes_{B} F^{\bullet}$ and $J^{\bullet \bullet}$ is an absolutely acyclic complex of fp-injective left $A$-modules, and the cone of the natural morphism from the complex $F^{\bullet}$ to the total complex of $\operatorname{Hom}_{A}\left(D^{\bullet}, J^{\bullet \bullet}\right)$ is an absolutely acyclic complex of flat left $B$-modules.

\section{Semiderived Categories and Relative Dualizing Complexes}

The aim of this section is to define the notion of a relative dualizing complex for a pair of homomorphisms of noncommutative rings $A \longrightarrow R$ and $B \longrightarrow S$, and obtain a related covariant equivalence between the semiderived categories of modules. One can say that a relative dualizing complex is "a dualizing complex in the direction of $A$ and $B$, and a dedualizing complex in the direction of $R$ relative to $A$ and $S$ relative to $B$ " (see the paper [19] for a discussion of dedualizing complexes). The resulting equivalence of semiderived categories resembles the derived semimodulesemicontramodule correspondence of [14, Sections 0.3.7 and 6.3].

The following definition is to be compared with those in [14, Sections 0.3.3 and 2.3]; see also [1, Proposition 2.1.1(2)]. Let $A \longrightarrow R$ be a morphism of associative rings. The $R / A$-semicoderived category $\mathrm{D}_{A}^{\text {sico }}(R$-mod) of left $R$-modules is defined as the quotient category of the homotopy category of left $R$-modules $\operatorname{Hot}(R$-mod) by its thick subcategory of complexes of $R$-modules that are coacyclic as complexes of $A$-modules. Similarly, assuming that the ring $A$ is left coherent, the $R / A$-semicoderived category of $A$-fp-injective left $R$-modules $\mathrm{D}_{A}^{\text {sico }}\left(R-\bmod _{A-\mathrm{fpi}}\right)$ is defined as the quotient category of the homotopy category of (complexes of) $A$-fp-injective left $R$-modules $\operatorname{Hot}\left(R-\bmod _{A-\mathrm{fpi}}\right)$ by its thick subcategory of complexes that are coacyclic in the exact category of fp-injective left A-modules.

The next definition is to be compared with those in [14, Sections 0.3.6 and 4.3]; see also [1, Proposition 2.1.1(1)]. Let $B \longrightarrow S$ be a morphism of associative rings. The $S / B$-semicontraderived category $\mathrm{D}_{B}^{\text {sictr }}(S-$-mod $)$ of left $S$-modules is defined as the quotient category of the homotopy category of left $S$-modules $\operatorname{Hot}(S-$ mod $)$ by 
its thick subcategory of complexes of $S$-modules that are contraacyclic as complexes of $B$-modules. Similarly, assuming that the $\operatorname{ring} B$ is right coherent, the $S / B$-semicontraderived category of $B$-flat left $S$-modules $\mathrm{D}_{B}^{\text {sictr }}\left(S-\bmod _{B-\mathrm{fl}}\right)$ is defined as the quotient category of the homotopy category of (complexes of) $B$-flat left $S$-modules $\operatorname{Hot}\left(S-\bmod _{B-\mathrm{fl}}\right)$ by its thick subcategory of complexes that are contraacyclic in the exact category of flat left $B$-modules.

Theorem 5.1. (a) Let $A \longrightarrow R$ be a morphism of associative rings; assume that the ring $A$ is left coherent and the ring $R$ is a flat right $A$-module. Then the triangulated functor between the semicoderived categories $\mathrm{D}_{A}^{\text {sico }}\left(R-\bmod _{A-\mathrm{fpi}}\right) \longrightarrow \mathrm{D}_{A}^{\text {sico }}(R-\mathrm{mod})$ induced by the embeddings of exact categories $A-\bmod _{\mathrm{fpi}} \longrightarrow A-\bmod$ and $R-\bmod _{A-\mathrm{fpi}} \longrightarrow$ $R$-mod is an equivalence of triangulated categories.

(b) Let $B \longrightarrow S$ be a morphism of associative rings; assume that the ring $B$ is right coherent and the ring $S$ is a flat left $B$-module. Then the triangulated functor between the semicontraderived categories $\mathrm{D}_{B}^{\text {sictr }}\left(S-\bmod _{B-\mathrm{fl}}\right) \longrightarrow \mathrm{D}_{B}^{\text {sictr }}(S-\bmod )$ induced by the embeddings of exact categories $B-\bmod _{\mathrm{fl}} \longrightarrow B-\bmod$ and $S-\bmod _{B-\mathrm{fl}} \longrightarrow S-\bmod$ is an equivalence of triangulated categories.

Proof. Part (a): in view of [16, Lemma 1.6], it suffices to show that for any complex of left $R$-modules $M \bullet$ there exists a complex of $A$-fp-injective left $R$-modules $J \bullet$ together with a morphism of complexes of $R$-modules $M^{\bullet} \longrightarrow J^{\bullet}$ with a cone coacyclic as a complex of left $A$-modules. Indeed, since $R$ is a flat right $A$-module, any injective left $R$-module is also an injective left $A$-module. Hence any complex of left $R$-modules $M^{\bullet}$ can be embedded into a complex of $A$-fp-injective left $R$-modules. The quotient complex can be also similarly embedded, etc. Totalizing the bicomplex constructed in this way by taking infinite direct sums along the diagonals, one obtains the desired complex of $A$-fp-injective left $R$-modules $J^{\bullet}$; the cone of the natural morphism $M^{\bullet} \longrightarrow J^{\bullet}$ is even coacyclic as a complex of left $R$-modules [14, Lemma 2.1].

Part (b): it suffices to show that for any complex of left $S$-modules $N^{\bullet}$ there exists a complex of $B$-flat left $S$-modules $F^{\bullet}$ together with a morphism of complexes of $S$-modules $F^{\bullet} \longrightarrow N^{\bullet}$ with a cone contraacyclic as a complex of left $B$-modules. Indeed, since $S$ is a flat left $B$-module, any flat left $S$-module is also flat over $B$. Hence any complex of left $S$-modules $N^{\bullet}$ is the image of a surjective morphism from a complex of $B$-flat left $S$-modules. The kernel can be also presented as such an image, etc. Totalizing the bicomplex constructed in this way by taking infinite products along the diagonals, one obtains the desired complex of $B$-flat left $S$-modules $F^{\bullet}$; the cone of the natural morphism $F^{\bullet} \longrightarrow N^{\bullet}$ is even contraacyclic as a complex of left $S$-modules [18, Section A.3].

Let us denote by $\mathrm{D}\left(R-\bmod _{A-\text { inj }}\right)$ the quotient category of the homotopy category of (complexes of) $A$-injective left $R$-modules by the thick subcategory of complexes that are contractible as complexes of left $A$-modules. Similarly, denote by $\mathrm{D}\left(S-\bmod _{B-\text { proj }}\right)$ the quotient category of the homotopy category of $B$-projective left $R$-modules by the thick subcategory of complexes that are contractible as complexes of left $B$-modules. Notice that the triangulated categories $\mathrm{D}\left(R-\bmod _{A-\text { inj }}\right)$ and $\mathrm{D}\left(S-\bmod _{B-\text { proj }}\right)$ are the 
conventional derived categories of the exact categories of $A$-injective left $R$-modules and $B$-projective left $S$-modules $R$ - $\bmod _{A \text {-inj }}$ and $S-\bmod _{B \text {-proj }}$ (the "coderived category along $A$ " and the "contraderived category along $B$ " tokens are expressed in the passages from the abelian category $R$-mod to its exact subcategory $R$ - $\bmod _{A \text {-inj }}$ and from the abelian category $S$-mod to its exact subcategory $S-\bmod _{B-\text { proj }}$ ).

The following result provides an interpretation of the semiderived categories $\mathrm{D}_{A}^{\text {sico }}(R$-mod $)$ and $\mathrm{D}_{B}^{\text {sictr }}(R-$ mod $)$ in the spirit of the definitions of the coderived and contraderived categories as "homotopy categories of complexes of injectives" and "homotopy categories of complexes of projectives" (as in $[6,7,8]$ and $[1,22]$ ).

Theorem 5.2. (a) Let $A \longrightarrow R$ be a morphism of associative rings; assume that the ring $A$ is left coherent, the ring $R$ is a flat right $A$-module, and all fp-injective left $A$-modules have finite injective dimensions. Then the triangulated functor between the (semico) derived categories $\mathrm{D}\left(R-\bmod _{A-\mathrm{inj}}\right) \longrightarrow \mathrm{D}_{A}^{\text {sico }}(R$-mod) induced by the embeddings of exact categories $A-\bmod _{\mathrm{inj}} \longrightarrow A-\bmod$ and $R-\bmod _{A-\mathrm{inj}} \longrightarrow R-\bmod$ is an equivalence of triangulated categories.

(b) Let $B \longrightarrow S$ be a morphism of associative rings; assume that the ring $B$ is right coherent, the ring $S$ is a projective left $B$-module, and all flat left $B$-modules have finite projective dimensions. Then the triangulated functor between the (semicontra)derived categories $\mathrm{D}\left(S-\bmod _{B-\text { proj }}\right) \longrightarrow \mathrm{D}_{B}^{\text {sictr }}(S-\bmod )$ induced by the embeddings of exact categories $B-\bmod _{\text {proj }} \longrightarrow B-\bmod$ and $S-\bmod _{B-\text { proj }} \longrightarrow S-\bmod$ is an equivalence of triangulated categories.

Proof. Part (a): in view of the construction in the proof of Theorem 5.1(a), it remains to show that for any complex of $A$-fp-injective left $R$-modules $J^{\bullet}$ there exists a complex of $A$-injective left $R$-modules $K^{\bullet}$ together with a morphism of complexes of $R$-modules $J^{\bullet} \longrightarrow K^{\bullet}$ with a cone coacyclic as a complex of $A$-modules. This is easily done using the finite resolution argument of [16, Sections 3.6-3.7] and [18, Section A.5]. The proof of part (b) is similar (cf. [16, Section 3.8]). One only has to notice that since the ring $S$ is a projective left $B$-module, any projective left $S$-module is also projective over $B$; so any complex of left $S$-modules is the image of a surjective morphism from a complex of $B$-projective left $S$-modules.

In order to formulate the derived semico-semicontra correspondence (noncommutative covariant relative Serre-Grothendieck duality) theorem, we need several more definitions. Let $A \longrightarrow R$ be a morphism of associative rings; assume that the ring $R$ is a flat right $A$-module. A left $R$-module $P$ is said to be weakly projective relative to $A$ (weakly $R / A$-projective) if the functor $\operatorname{Hom}_{R}(P,-)$ takes short exact sequences of $A$-injective left $R$-modules to short exact sequence of abelian groups (cf. [15, Sections 4.1 and 4.3] and [14, Sections 5.1.4, 5.3 and 9.1]). Similarly, let $B \longrightarrow S$ be a morphism of associative rings; assume that the ring $S$ is a flat left $B$-module. A right $S$-module $F$ is said to be weakly flat relative to $B$ (weakly $S / B$-flat) if the functor $F \otimes_{S}$ - takes short exact sequences of $B$-flat left $S$-modules to short exact sequences of abelian groups (cf. [14, Section 5.1.6]). 
Lemma 5.3. (a) A left $R$-module $P$ is weakly $R / A$-projective if and only if $\operatorname{Ext}_{R}^{1}(P, J)=0$ for any $A$-injective left $R$-module $J$, and if and only if $\operatorname{Ext}_{R}^{n}(P, J)=0$ for all $n>0$ and any such $J$. Consequently, the class of weakly $R / A$-projective left $R$-modules is closed under extensions and the passages to the kernels of surjective morphisms.

(b) A right $S$-module $F$ is weakly $S / B$-flat if and only if $\operatorname{Tor}_{1}^{R}(F, G)=0$ for any $B$-flat left $S$-module $G$, and if and only if $\operatorname{Tor}_{n}^{R}(F, G)=0$ for all $n>0$ and any such $G$. Consequently, the class of weakly $S / B$-flat right $S$-modules is closed under extensions and the passages to the kernels of surjective morphisms.

Proof. Part (a): it is clear that any left $R$-module $P$ satisfying the Ext $^{1}$ vanishing condition satisfies the definition of weakly relative projectivity. In order to show that $\operatorname{Ext}_{R}^{>0}(P, J)=0$ for any weakly $R / A$-projective left $R$-module $P$ and $A$-injective left $R$-module $J$, one simply notices that any injective right resolution of the $R$-module $J$ is exact with respect to the exact category of $A$-injective left $R$-modules (since injective left $R$-modules are $A$-injective). Part (b): it is clear that any right $S$-module $F$ satisfying the Tor $_{1}$ vanishing condition satisfies the definition of weakly relative flatness. To check that $\operatorname{Tor}_{>0}^{R}(F, G)=0$ for any weakly $S / B$-flat right $S$-module $F$ and any $B$-flat left $S$-module $G$, one notices that any flat left resolution of the $S$-module $G$ is exact with respect to the exact category of $B$-flat left $S$-modules (since flat left $S$-modules are $B$-flat).

Here is the main definition of this section. Let $A \longrightarrow R$ and $B \longrightarrow S$ be a pair of associative ring homomorphisms; assume that the $\operatorname{ring} A$ is left coherent, the ring $B$ is right coherent, the ring $R$ is a flat right $A$-module, and the ring $S$ is a flat left $B$-module. A relative dualizing complex for the pair of morphisms $A \longrightarrow R$ and $B \longrightarrow S$ is a triple consisting of a dualizing complex $D^{\bullet}$ for the rings $A$ and $B$, a finite complex of $R$-S-bimodules $T^{\bullet}$, and a morphism of complexes of $A$ - $B$-bimodules $D^{\bullet} \longrightarrow T^{\bullet}$ satisfying the following conditions:

(iv) the terms of the complex $T^{\bullet}$ are weakly $R / A$-projective left $R$-modules and weakly $S / B$-flat right $S$-modules;

(v) the morphism of complexes of $R$-B-bimodules $R \otimes_{A} D^{\bullet} \longrightarrow T^{\bullet}$ and the morphism of complexes of $A$-S-bimodules $D^{\bullet} \otimes_{B} S \longrightarrow T^{\bullet}$ induced by the morphism $D^{\bullet} \longrightarrow T^{\bullet}$ are quasi-isomorphisms of finite complexes.

Example 5.4. (1) Let $A$ and $B$ be associative algebras over a commutative ring $k$; assume that the ring $A$ is left coherent and the $\operatorname{ring} B$ is right coherent. Suppose that a dualizing complex $D^{\bullet}$ for the rings $A$ and $B$ is a complex of $A$-B-bimodules over $k$ (i. e., the left and right $k$-module structures on $D^{\bullet}$ coincide). Let $U$ be a $k$-flat associative algebra over $k$. Consider the natural homomorphisms of associative rings $A \longrightarrow U \otimes_{k} A=R$ and $B \longrightarrow U \otimes_{k} B=S$. Then the complex of $R$-S-bimodules $T^{\bullet}=U \otimes_{k} D^{\bullet}$ together with the natural morphism $D^{\bullet} \longrightarrow T^{\bullet}$ is a relative dualizing complex for the pair of ring homomorphisms $A \longrightarrow R$ and $B \longrightarrow S$.

(2) In particular, let $A$ be a coherent commutative ring and $R$ be an $A$-flat associative $A$-algebra. Let $D^{\bullet}$ be a dualizing complex for the $\operatorname{ring} A$ (i. e., a complex of 
$A$-modules that, viewed as a complex of $A$ - $A$-bimodules, is a dualizing complex for the rings $A$ and $A$ ). Then the complex of $R$ - $R$-bimodules $T^{\bullet}=R \otimes_{A} D^{\bullet}$ together with the natural morphism $D^{\bullet} \longrightarrow T^{\bullet}$ is a relative dualizing complex for the pair of ring homomorphisms $A \longrightarrow R$ and $A \longrightarrow R$.

Example 5.5. (1) Let $A \longrightarrow R$ be a homomorphism of associative algebras over a field $k$ such that the algebra $A$ is finite-dimensional and the ring $R$ is a projective right $A$-module. Then the tensor product $R \otimes_{A} A^{*}$ has a natural structure of a semialgebra over the coalgebra $A^{*}\left[14\right.$, Section 10.2.1]. The $A$ - $A$-bimodule $R \otimes_{A} A^{*}$ is an injective right $A$-module by construction; suppose further that it is an injective left $A$-module. Set $S=\operatorname{Hom}_{A}\left(A^{*}, R \otimes_{A} A^{*}\right)$; the $A$-A-bimodule $S$ can be also defined as the cotensor product $A \square_{A^{*}}\left(R \otimes_{A} A^{*}\right)$ over the coalgebra $A^{*}$. Then there is a natural associative algebra structure on $S$ and a natural homomorphism of associative algebras $A \longrightarrow S\left[14\right.$, Section B.2.2]. The $R$-S-bimodule $R \otimes_{A} A^{*}=\mathcal{T} \simeq A^{*} \otimes_{A} S$, together with the natural map $A^{*} \longrightarrow \mathcal{T}$, is a relative dualizing complex for the pair of ring homomorphisms $A \longrightarrow R$ and $A \longrightarrow S$.

(2) More generally, let $K$ be a classically semisimple ring and $K \longrightarrow A \longrightarrow R$ be associative ring homomorphisms such that $A$ is a finitely generated projective left $K$-module and $R$ is a projective right $A$-module. Set $\mathcal{C}=\operatorname{Hom}_{K}(A, K)$ and suppose that $\mathcal{C}$ is a finitely generated projective left $K$-module (see Example 3.3 above). Set $B=\operatorname{Hom}_{K}(\mathcal{C}, K)$. The $A$-B-bimodule $R \otimes_{A} \mathcal{C}$ is an injective right $B$-module by construction; suppose further that it is an injective left $A$-module. Set $S=\operatorname{Hom}_{A}\left(\mathrm{C}, R \otimes_{A} \mathrm{C}\right)$; the $B$ - $B$-bimodule $S$ can be also defined as the cotensor product $B \square_{\mathcal{C}}\left(R \otimes_{A} \mathcal{C}\right)$ over the coring $\mathcal{C}$.

The tensor product $R \otimes_{A} \mathcal{C}$ has a natural structure of a semialgebra over the coring $\mathcal{C}$. There is a natural associative ring structure on $S$ and a natural homomorphism of associative rings $B \longrightarrow S$ [15, Section 3.3]. The $R$-S-bimodule $R \otimes_{A} \mathrm{C}=\mathfrak{T} \simeq \mathcal{C} \otimes_{B} S$, together with the natural map $\mathrm{C} \longrightarrow \mathcal{T}$, is a relative dualizing complex for the pair of ring homomorphisms $A \longrightarrow R$ and $B \longrightarrow S$.

The following theorem is our main result.

Theorem 5.6. Let $A \longrightarrow R$ and $B \longrightarrow S$ be a pair of associative ring homomorphisms; assume that the ring $A$ is left coherent, the ring $B$ is right coherent, the ring $R$ is a flat right $A$-module, the ring $S$ is a flat left $B$-module, and all fp-injective left A-modules have finite injective dimensions. Let $D^{\bullet} \longrightarrow T^{\bullet}$ be a relative dualizing complex for the pair of morphisms $A \longrightarrow R$ and $B \longrightarrow S$. Then there is an equivalence between the $R / A$-semicoderived category of left $R$-modules $\mathrm{D}_{A}^{\text {sico }}(R$-mod) and the $S / B$-semicontraderived category of left $S$-modules $\mathrm{D}_{B}^{\text {sictr }}(S$-mod $)$ provided by the mutually inverse functors $\mathbb{R} \operatorname{Hom}_{R}\left(T^{\bullet},-\right)$ and $T^{\bullet} \otimes_{S}^{\mathbb{L}}-$.

Proof. The derived functor $\mathbb{R} \operatorname{Hom}_{R}\left(T^{\bullet},-\right): \mathrm{D}_{A}^{\text {sico }}(R$-mod $) \longrightarrow \mathrm{D}_{B}^{\text {sictr }}(S$-mod $)$ is constructed by identifying the semicoderived category $\mathrm{D}_{A}^{\text {sico }}(R-$ mod $)$ with the derived category $\mathrm{D}\left(R-\bmod _{A \text {-inj }}\right)$ (see Theorem 5.2(a)) and applying the functor $\operatorname{Hom}_{R}\left(T^{\bullet},-\right)$ to complexes of $A$-injective left $R$-modules. Given a complex of $A$-injective left 
$R$-modules $J^{\bullet}$, there is a natural morphism of complexes of left $B$-modules

$$
\operatorname{Hom}_{R}\left(T^{\bullet}, J^{\bullet}\right) \longrightarrow \operatorname{Hom}_{R}\left(R \otimes_{A} D^{\bullet}, J^{\bullet}\right) \simeq \operatorname{Hom}_{A}\left(D^{\bullet}, J^{\bullet}\right) ;
$$

in view of Lemma 5.3(a), the cone of this morphism is the total complex of a finite acyclic complex of complexes of left $B$-modules, that is an absolutely acyclic complex of left $B$-modules. In particular, it follows that the complex of left $S$-modules $\operatorname{Hom}_{R}\left(T^{\bullet}, J^{\bullet}\right)$ is $B$-contraacyclic whenever a complex of $A$-injective left $R$-modules $J^{\bullet}$ is contractible as a complex of left $A$-modules.

The derived functor $T^{\bullet} \otimes_{S}^{\mathbb{L}}-: \mathrm{D}_{B}^{\text {sictr }}(S-$ mod $) \longrightarrow \mathrm{D}_{A}^{\text {sico }}(R-$ mod $)$ is obtained by identifying the semicontraderived category $\mathrm{D}_{B}^{\text {sictr }}(S-$ mod $)$ with the semicontraderived category $D_{B}^{\text {sictr }}\left(S-\bmod _{B-\mathrm{fl}}\right)$ (see Theorem $\left.5.1(\mathrm{~b})\right)$ and applying the functor $T^{\bullet} \otimes_{S}-$ to complexes of $B$-flat left $S$-modules. Notice that, by Proposition 4.3, the exact category of flat left $B$-modules has finite homological dimension, so any contraacyclic complex of flat left $B$-modules is absolutely acyclic with respect to the exact category $B$ - $\bmod _{\mathrm{fl}}$. Given a complex of $B$-flat left $S$-modules $G^{\bullet}$, there is a natural morphism of complexes of left $A$-modules

$$
D^{\bullet} \otimes_{B} G^{\bullet} \simeq\left(D^{\bullet} \otimes_{B} S\right) \otimes_{S} G^{\bullet} \longrightarrow T^{\bullet} \otimes_{S} G^{\bullet}
$$

in view of Lemma $5.3(\mathrm{~b})$, the cone of this morphism is the total complex of a finite acyclic complex of complexes of left $A$-modules, that is an absolutely acyclic complexes of left $A$-modules. It follows that the complex of left $R$-modules $T^{\bullet} \otimes_{S} G^{\bullet}$ is $A$-coacyclic whenever a complex of $B$-flat left $S$-modules $G^{\bullet}$ is $B$-contraacyclic.

We have constructed the derived functors $\mathbb{R} \operatorname{Hom}_{R}\left(T^{\bullet},-\right): \mathrm{D}_{A}^{\text {sico }}(R-$ mod $) \longrightarrow$ $\mathrm{D}_{B}^{\text {sictr }}(S-$ mod $)$ and $T^{\bullet} \otimes_{S}^{\mathbb{L}}-: \mathrm{D}_{B}^{\text {sictr }}(S-$ mod $) \longrightarrow \mathrm{D}_{A}^{\text {sico }}(R-$ mod $)$. It is easy to show that the former of them is right adjoint to the latter (see, e. g., [14, Lemma 8.3]). It remains to check that the adjunction morphisms are isomorphisms in $\mathrm{D}_{A}^{\text {sico }}(R$-mod $)$ and $\mathrm{D}_{B}^{\mathrm{sictr}}(S-\mathrm{mod})$. Now we recall that, as we have just seen, the forgetful functors

$$
\mathrm{D}_{A}^{\text {sico }}(R-\bmod ) \longrightarrow \mathrm{D}^{\mathrm{co}}(A-\bmod )
$$

and

$$
\mathrm{D}_{B}^{\text {sictr }}(S-\bmod ) \longrightarrow \mathrm{D}^{\mathrm{ctr}}(B-\mathrm{mod})
$$

transform the functor $\mathbb{R} \operatorname{Hom}_{R}\left(T^{\bullet},-\right)$ into the functor $\mathbb{R} \operatorname{Hom}_{A}\left(D^{\bullet},-\right)$ and the functor $T^{\bullet} \otimes_{S}^{\mathbb{L}}$ - into the functor $D^{\bullet} \otimes_{B}^{\mathbb{L}}-$, i. e., there are commutative diagrams of triangulated functors

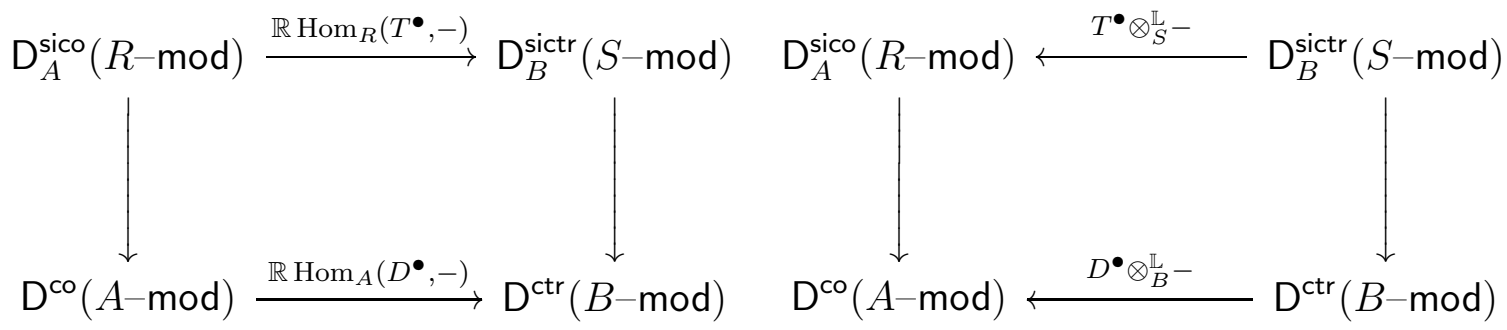


The forgetful functors also transform the adjunction morphisms for the pair of functors $\mathbb{R} \operatorname{Hom}_{R}\left(T^{\bullet},-\right)$ and $T^{\bullet} \otimes_{S}^{\mathbb{L}}$ - into the adjunction morphisms for the pair of functors $\mathbb{R} \operatorname{Hom}_{A}\left(D^{\bullet},-\right)$ and $D^{\bullet} \otimes_{B}^{\mathbb{L}}-$. Since the latter pair of adjunction morphisms are isomorphisms by Theorem 4.5 and the forgetful functors are conservative, the former pair of adjunction morphisms are isomorphisms, too.

\section{The Semitensor Product}

The coderived category of quasi-coherent sheaves on a separated Noetherian scheme with a dualizing complex has a tensor category structure depending on the choice of a dualizing complex, which is the unit object of this tensor category structure (see [13, Chapter 6, Proposition 8.10, and Appendix B] or [17, Section B.2.5]). The aim of this section is to extend this construction from Noetherian to coherent commutative rings, and further to the relative situation with a flat commutative algebra $R$ over a coherent commutative ring $A$ with a dualizing complex $D^{\bullet}$. The tensor category structure with the unit object $T^{\bullet}=R \otimes_{A} D^{\bullet}$ will be defined on the $R / A$-semicoderived category $\mathrm{D}_{A}^{\text {sico }}(R$-mod $)$ in the relative situation.

The construction is based on the results of Sections 4-5. As in these sections, we will have to make the technical assumption that fp-injective $A$-modules have finite injective dimensions. Throughout this section, the term dualizing complex of $A$-modules is understood in the sense of Example 5.4(2); i. e., it is a complex of $A$-modules which, viewed as a complex of $A$ - $A$-bimodules, is a dualizing complex for the rings $A$ and $A$, in the sense of the definition in Section 4.

Theorem 6.1. Let $A$ be a coherent commutative ring such that fp-injective $A$-modules have finite injective dimensions, and let $D^{\bullet}$ be a dualizing complex of A-modules. Then there is a natural associative, commutative, and unital tensor category structure on the coderived category of A-modules, provided by the operation of (derived) cotensor product of complexes of $A$-modules

$$
\square_{D^{\bullet}}: \mathrm{D}^{\mathrm{co}}(A-\bmod ) \times \mathrm{D}^{\mathrm{co}}(A-\bmod ) \longrightarrow \mathrm{D}^{\mathrm{co}}(A-\bmod ) .
$$

The dualizing complex $D^{\bullet} \in \mathrm{D}_{\mathrm{fp}}^{\mathrm{b}}(A-\mathrm{mod}) \subset \mathrm{D}^{\mathrm{co}}(A$-mod $)$ is the unit object of this tensor category structure.

Proof. We use the results of Section 4 in order to identify the coderived category of $A$-modules $\mathrm{D}^{\mathrm{co}}(A$-mod $)$ with the absolute derived category of flat $A$-modules $\mathrm{D}^{\text {abs }}\left(A-\bmod _{\mathrm{fl}}\right)$. Indeed, by Theorem 4.5 , the choice of a dualizing complex $D^{\bullet}$ induces an equivalence between the coderived category and the contraderived category of $A$-modules, $\mathrm{D}^{\mathrm{co}}(A-$ mod $) \simeq \mathrm{D}^{\mathrm{ctr}}(A-$ mod $)$, and by Theorem $4.4(\mathrm{a})$, the contraderived category of $A$-modules is equivalent to the contraderived category of flat $A$-modules, $\mathrm{D}^{\mathrm{ctr}}(A-\bmod ) \simeq \mathrm{D}^{\mathrm{ctr}}\left(A-\bmod _{\mathrm{fl}}\right)$. By Proposition 4.3 , flat $A$-modules have finite projective dimensions, and by [14, Remark 2.1], it follows that contraacyclic complexes of flat $A$-modules are absolutely acyclic with respect to the exact category of flat $A$-modules, so the contraderived and the absolute derived categories of flat $A$-modules 
coincide, $\mathrm{D}^{\mathrm{ctr}}\left(A-\bmod _{\mathrm{fl}}\right)=\mathrm{D}^{\mathrm{abs}}\left(A-\bmod _{\mathrm{fl}}\right)$ (see [18, Section A.1] for the definition of the absolute derived category).

Notice that the tensor product of a complex of flat $A$-modules and an absolutely acyclic complex of flat $A$-modules is obviously an absolutely acyclic complex of flat $A$-modules, so there is the tensor product functor

$$
\otimes_{A}: \mathrm{D}^{\mathrm{abs}}\left(A-\bmod _{\mathrm{fl}}\right) \times \mathrm{D}^{\mathrm{abs}}\left(A-\bmod _{\mathrm{fl}}\right) \longrightarrow \mathrm{D}^{\mathrm{abs}}\left(A-\bmod _{\mathrm{fl}}\right) .
$$

By the definition, the cotensor product functor $\square_{D}$ • is obtained from this functor of tensor product of complexes of flat $A$-modules using the equivalence of categories $\mathrm{D}^{\mathrm{co}}(A-\bmod ) \simeq \mathrm{D}^{\text {abs }}\left(A-\bmod _{\mathrm{fl}}\right)$. Explicitly, this means that in order to compute the cotensor product of two objects of the coderived category, one has to represent them by two complexes of injective $A$-modules $I^{\bullet}$ and $J^{\bullet}$ (using the result of Theorem 2.4) and then apply the formula

$$
I^{\bullet} \square_{D^{\bullet}} J^{\bullet}=D^{\bullet} \otimes_{A} \operatorname{Hom}_{A}\left(D^{\bullet}, I^{\bullet}\right) \otimes_{A} \operatorname{Hom}_{A}\left(D^{\bullet}, J^{\bullet}\right) .
$$

Furthermore, there is a well-defined functor of tensor product of complexes of flat $A$-modules and arbitrary complexes of $A$-modules

$$
\otimes_{A}: \mathrm{D}^{\mathrm{abs}}\left(A-\bmod _{\mathrm{fl}}\right) \times \mathrm{D}^{\mathrm{co}}(A-\bmod ) \longrightarrow \mathrm{D}^{\mathrm{co}}(A-\bmod ),
$$

since the tensor product of an absolutely acyclic complex of flat $A$-modules with any complex of $A$-modules is a coacyclic (in fact, absolutely acyclic) complex of $A$-modules, as is the tensor product of a complex of flat $A$-modules with a coacyclic complex of $A$-modules. The cotensor product of two complexes of injective $A$-modules can be alternatively defined by the rules

$$
I^{\bullet} \square_{D^{\bullet}} J^{\bullet} \simeq \operatorname{Hom}_{A}\left(D^{\bullet}, I^{\bullet}\right) \otimes_{A} J^{\bullet} \simeq I^{\bullet} \otimes_{A} \operatorname{Hom}_{A}\left(D^{\bullet}, J^{\bullet}\right),
$$

where the isomorphism signs denote natural isomorphisms in the absolute derived category of fp-injective $A$-modules (cf. [13, Proposition 8.10] and [17, Section B.2.5]).

The following theorem is the main result of this section.

Theorem 6.2. Let $A$ be a coherent commutative ring such that fp-injective A-modules have finite injective dimensions, and let $D^{\bullet}$ be a dualizing complex of A-modules. Let $A \longrightarrow R$ be a morphism of commutative rings making $R$ a flat $A$-module. Then there is a natural associative, commutative, and unital tensor category structure on the $R / A$-semicoderived category of $R$-modules, provided by the functor of (derived) semitensor product of complexes of $R$-modules

$$
\diamond_{T} \bullet: \mathrm{D}_{A}^{\text {sico }}(R-\bmod ) \times \mathrm{D}_{A}^{\text {sico }}(R-\bmod ) \longrightarrow \mathrm{D}_{A}^{\text {sico }}(R-\bmod ),
$$

where $T^{\bullet}=R \otimes_{A} D^{\bullet}$ is the relative dualizing complex for the ring homomorphism $A \longrightarrow R$ associated with the dualizing complex $D^{\bullet}$ for the ring $A$. The relative dualizing complex $T^{\bullet} \in \mathrm{D}_{A}^{\text {sico }}(R$-mod) is the unit object of this tensor category structure. 
Proof. To constuct the functor $\diamond_{T}$, we will use the results of Section 5 in order to identify the semicoderived category $\mathrm{D}_{A}^{\text {sico }}(R$-mod) with the quotient category of homotopy category of complexes of $A$-flat $R$-modules by its thick subcategory of complexes that are absolutely acyclic as complexes of flat $A$-modules. Let us call the latter category simply the semiderived or the $R / A$-semiderived category of $A$-flat $R$-modules and denote it by $\mathrm{D}_{A}^{\mathrm{si}}\left(R-\bmod _{A-\mathrm{fl}}\right)$.

Indeed, by Theorem 5.6, the choice of the relative dualizing complex $D^{\bullet} \longrightarrow$ $T^{\bullet}$ for a ring homomorphism $A \longrightarrow R$ induces an equivalence between the $R / A$-semicoderived category and the $R / A$-semicontraderived category of $R$-modules, $\mathrm{D}_{A}^{\text {sico }}(R$-mod $) \simeq \mathrm{D}_{A}^{\text {sictr }}(R$-mod $)$. By Theorem 5.1(b), the $R / A$-semicontraderived category of $R$-modules is equivalent to the $R / A$-semicontraderived category of $A$-flat $R$-modules, $\mathrm{D}_{A}^{\text {sictr }}(R$-mod $) \simeq \mathrm{D}_{A}^{\text {sictr }}\left(R-\bmod _{A-\mathrm{fl}}\right)$, and since the exact category of flat $A$-modules has finite homological dimension, the $R / A$-semicontraderived category of $A$-flat $R$-modules coincides with their $R / A$-semiderived category, $\mathrm{D}_{A}^{\text {sictr }}\left(R-\bmod _{A-\mathrm{fl}}\right)=\mathrm{D}_{A}^{\mathrm{si}}\left(R-\bmod _{A-\mathrm{fl}}\right)$.

Composing these equivalences, we obtain an equivalence of triangulated categories $\mathrm{D}_{A}^{\text {si }}\left(R-\bmod _{A-\mathrm{fl}}\right) \simeq \mathrm{D}_{A}^{\text {sico }}(R$-mod $)$ provided by the functor assigning to a complex of $A$-flat $R$-modules $G^{\bullet}$ the complex of $R$-modules $T^{\bullet} \otimes_{R} G^{\bullet} \simeq D^{\bullet} \otimes_{A} G^{\bullet}$. The inverse functor assigns to a complex of $A$-injective $R$-modules $J^{\bullet}$ the complex of $A$-flat $R$-modules $\operatorname{Hom}_{R}\left(T^{\bullet}, J^{\bullet}\right) \simeq \operatorname{Hom}_{A}\left(D^{\bullet}, J^{\bullet}\right)$.

Furthermore, it will be more convenient for us to work with the $R / A$-semicoderived category of $A$-flat $R$-modules $\mathrm{D}^{\text {sico }}\left(R\right.$ - $\left.\bmod _{A-\mathrm{fl}}\right)$, defined as the quotient category of the homotopy category of $A$-flat $R$-modules by its thick subcategory of complexes that are coacyclic as complexes of flat A-modules. Since, in view of Proposition 4.3, the exact category of flat $A$-modules has finite projective dimension, by $[14$, Remark 2.1] the $R / A$-semiderived category of $A$-flat $R$-modules coincides with their $R / A$-semicoderived category, $\mathrm{D}_{A}^{\mathrm{si}}\left(R-\bmod _{A-\mathrm{fl}}\right)=\mathrm{D}_{A}^{\text {sico }}\left(R-\bmod _{A-\mathrm{fl}}\right)$.

Let us emphasize that while, by Theorem 4.4(a), any complex of flat $A$-modules that is contraacyclic as a complex of $A$-modules is also contraacyclic as a complex of flat $A$-modules, there is no claim that a complex of flat $A$-modules coacyclic as a complex of $A$-modules should be coacyclic as a complex of flat $A$-modules (cf. [13, Chapter 3] and [17, Remark 1.5 and Section 2.5]). Whenever below we mention a "coacyclic complex of flat $A$-modules", it means a complex coacyclic with respect to the exact category of flat $A$-modules.

Proposition 6.3. Let $A \longrightarrow R$ be an arbitrary morphism of commutative rings such that $R$ is a flat $A$-module. Then one can construct the left derived functor of tensor product of complexes of $A$-flat $R$-modules

$$
\otimes_{R}^{\mathbb{L}}: \mathrm{D}_{A}^{\text {sico }}\left(R-\bmod _{A-\mathrm{fl}}\right) \times \mathrm{D}_{A}^{\text {sico }}\left(R-\bmod _{A-\mathrm{fl}}\right) \longrightarrow \mathrm{D}_{A}^{\text {sico }}\left(R-\bmod _{A-\mathrm{fl}}\right)
$$

providing an associative, commutative, and unital tensor category structure on the $R / A$-semicoderived category of $A$-flat $R$-modules $\mathrm{D}_{A}^{\text {sico }}\left(R-\bmod _{A-\mathrm{fl}}\right)$ with the unit object $R \in \mathrm{D}_{A}^{\text {sico }}\left(R-\bmod _{A-\mathrm{fl}}\right)$. 
In addition, one can construct the left derived functor of tensor product of complexes of $A$-flat $R$-modules and arbitrary complexes of $R$-modules

$$
\otimes_{R}^{\mathbb{L}}: \mathrm{D}_{A}^{\text {sico }}\left(R-\bmod _{A-\mathrm{fl}}\right) \times \mathrm{D}_{A}^{\text {sico }}(R-\bmod ) \longrightarrow \mathrm{D}_{A}^{\text {sico }}(R-\bmod )
$$

providing an associative and unital module category structure over the tensor category $\mathrm{D}_{A}^{\text {sico }}\left(R-\bmod _{A-\mathrm{fl}}\right)$ on the $R / A$-semicoderived category of $R$-modules $\mathrm{D}_{A}^{\text {sico }}(R$-mod $)$.

When $A$ is a coherent ring such that fp-injective $A$-modules have finite injective dimensions and a dualizing complex $D^{\bullet}$ is chosen for the ring $A$, the equivalence of categories $\mathrm{D}_{A}^{\text {sico }}\left(R-\bmod _{A-\mathrm{fl}}\right) \simeq \mathrm{D}_{A}^{\text {sico }}(R$-mod $)$ will transform both of the left derived functors of Proposition 6.3 into the desired functor of semitensor product $\diamond_{T} \bullet$.

Proof of Proposition 6.3. In order to obtain the derived functors $\otimes_{R}^{\mathbb{L}}$, we will apply the general construction of balanced derived functors of functors of two arguments elaborated in [14, Lemma 2.7]. Let us call a complex of $A$-flat $R$-modules $F^{\bullet}$ relatively homotopy $R$-flat if for any complex of $A$-flat $R$-modules $L^{\bullet}$ that is coacyclic as a complex of flat $A$-modules the complex of $R$-modules $F^{\bullet} \otimes_{R} L^{\bullet}$ is coacyclic as a complex of flat $A$-modules, and for any complex of $R$-modules $N^{\bullet}$ that is coacyclic as a complex of $A$-modules the complex of $R$-modules $F^{\bullet} \otimes_{R} N^{\bullet}$ is coacyclic as a complex of $A$-modules.

Similarly, let us call a complex of $R$-modules $H^{\bullet}$ homotopy $R / A$-flat if for any complex of $A$-flat $R$-modules $M^{\bullet}$ that is coacyclic as a complex of flat $A$-modules the complex of $R$-modules $M^{\bullet} \otimes_{R} H^{\bullet}$ is coacyclic as a complex of $A$-modules. Both the relatively homotopy $R$-flat complexes of $A$-flat $R$-modules and the homotopy $R / A$-flat complexes of $R$-modules are thought of as "homotopy flat in the direction of $R$ relative to $A$ " (while the former are supposed to be also complexes of flat $A$-modules, and the latter ones are not).

Lemma 6.4. Let $A \longrightarrow R$ be a morphism of commutative rings making $R$ a flat A-module. Then

(a) for any complex of $A$-flat $R$-modules $M^{\bullet}$ there exists a relatively homotopy $R$-flat complex of $A$-flat $R$-modules $F^{\bullet}$ together with a morphism of complexes of $R$-modules $F^{\bullet} \longrightarrow M^{\bullet}$ whose cone is coacyclic as a complex of flat A-modules;

(b) for any complex of $R$-modules $N^{\bullet}$ there exists a homotopy $R / A$-flat complex of $R$-modules $H^{\bullet}$ together with a morphism of complexes of $R$-modules $H^{\bullet} \longrightarrow N^{\bullet}$ whose cone is coacyclic as a complex of A-modules.

Proof. Part (a): notice that the full subcategory of relatively homotopy $R$-flat complexes of $A$-flat $R$-modules is closed under the operations of shift, cone, and the passage to an infinite direct sum (and consequently, also to the countable homotopy direct limit) in the homotopy category of complexes of $A$-flat $R$-modules $\operatorname{Hot}\left(R-\bmod _{A-\mathrm{fl}}\right)$. Besides, any complex of $R$-modules $R \otimes_{A} E^{\bullet}$ induced from a complex of flat $A$-modules $E^{\bullet}$ is a relatively homotopy $R$-flat complex of $A$-flat $R$-modules. Indeed, the tensor product of a complex of flat $A$-modules with a coacyclic complex of flat $A$-modules is a coacyclic complex of flat $A$-modules, and 
the tensor product of a complex of flat $A$-modules with a coacyclic complex of $A$-modules is a coacyclic complex of $A$-modules.

Now, given a complex of $A$-flat $R$-modules $M^{\bullet}$, consider the bar-complex

$$
\cdots \longrightarrow R \otimes_{A} R \otimes_{A} R \otimes_{A} M^{\bullet} \longrightarrow R \otimes_{A} R \otimes_{A} M^{\bullet} \longrightarrow R \otimes_{A} M^{\bullet} .
$$

Let $F^{\bullet}$ be the total complex of this bicomplex obtained by taking infinite direct sums along the diagonals. On the one hand, the complex $F^{\bullet}$ is homotopy equivalent to the homotopy direct limit of the total complexes of the finite segments (subcomplexes of silly filtration) of the bar-bicomplex, which are obtained from complexes of $R$-modules induced from complexes of flat $A$-modules by finite iterations of the operations of shift and cone. So the complex $F^{\bullet}$ is a relatively homotopy $R$-flat complex of $A$-flat $R$-modules. On the other hand, the cone of the natural morphism $F^{\bullet} \longrightarrow M^{\bullet}$ is not only coacyclic, but even contractible as a complex of (flat) $A$-modules.

Part (b): the full subcategory of homotopy $R / A$-flat complexes of $R$-modules is closed under the operations of shift, cone, and the passage to an infinite direct sum in the homotopy category of complexes of $R$-modules $\operatorname{Hot}(R$-mod). Besides, any complex of $R$-modules $R \otimes_{A} C^{\bullet}$ induced from a complex of $A$-modules $C^{\bullet}$ is homotopy $R / A$-flat. Indeed, the tensor product of a coacyclic complex of flat $A$-modules with any complex of $A$-modules is a coacyclic complex of $A$-modules.

Now, given a complex of $R$-modules $N^{\bullet}$, consider the bar-complex

$$
\cdots \longrightarrow R \otimes_{A} R \otimes_{A} R \otimes_{A} N^{\bullet} \longrightarrow R \otimes_{A} R \otimes_{A} N^{\bullet} \longrightarrow R \otimes_{A} N^{\bullet}
$$

Let $H^{\bullet}$ be the total complex of this bicomplex obtained by taking infinite direct sums along the diagonals. On the one hand, the complex $H^{\bullet}$ is homotopy equivalent to the homotopy direct limit of the subcomplexes of silly filtration on the bar-bicomplex, which are obtained from complexes of $R$-modules induced from complexes of $A$-modules by finite iterations of the operations of shift and cone. So $H^{\bullet}$ is a homotopy $R / A$-flat complex of $R$-modules. On the other hand, the cone of the natural morphism $H^{\bullet} \longrightarrow N^{\bullet}$ is a contractible complex of $A$-modules. (Cf. [14, Theorem 2.6].)

Now we can finish the proof of Proposition 6.3. Given two complexes of $A$-flat $R$-modules $M^{\bullet}$ and $N^{\bullet}$, one replaces one or both of them with relatively homotopy $R$-flat complexes of $A$-flat $R$-modules $F^{\bullet}$ and/or $G^{\bullet}$ endowed with morphisms of complexes of $R$-modules $F^{\bullet} \longrightarrow M^{\bullet}$ and $G^{\bullet} \longrightarrow N^{\bullet}$ with the cones coacyclic as complexes of flat $A$-modules. The induced morphisms of complexes of $A$-flat $R$-modules $F^{\bullet} \otimes_{R} G^{\bullet} \longrightarrow F^{\bullet} \otimes_{R} N^{\bullet}$ and $F^{\bullet} \otimes_{R} G^{\bullet} \longrightarrow M^{\bullet} \otimes_{R} G^{\bullet}$ have cones coacyclic with respect to the exact category of flat $A$-modules, so either of the three complexes $F^{\bullet} \otimes_{R} N^{\bullet}, M^{\bullet} \otimes_{R} G^{\bullet}$, or $F^{\bullet} \otimes_{R} G^{\bullet}$ can be taken as representing the object $M^{\bullet} \otimes_{R}^{\mathbb{L}} N^{\bullet}$ in the semicoderived category $\mathrm{D}_{A}^{\text {sico }}\left(R-\bmod _{A-\mathrm{fl}}\right)$.

Similarly, given a complex of $A$-flat $R$-modules $M^{\bullet}$ and an arbitrary complex of $R$-modules $N^{\bullet}$, one either replaces $M^{\bullet}$ with a relatively homotopy $R$-flat complex of $A$-flat $R$-modules $F^{\bullet}$ endowed with a morphism of complexes of $R$-modules $F^{\bullet} \longrightarrow$ $M^{\bullet}$ with the cone coacyclic as a complex of flat $A$-modules, or replaces $N^{\bullet}$ with a homotopy $R / A$-flat complex of $R$-modules $H^{\bullet}$ endowed with a morphism of complexes 
of $R$-modules $H^{\bullet} \longrightarrow N^{\bullet}$ with the cone coacyclic as a complex of $A$-modules. The induced morphisms of complexes of $R$-modules $F^{\bullet} \otimes_{R} H^{\bullet} \longrightarrow F^{\bullet} \otimes_{R} N^{\bullet}$ and $F^{\bullet} \otimes_{R}$ $H^{\bullet} \longrightarrow M^{\bullet} \otimes_{R} H^{\bullet}$ have cones coacyclic over $A$, so any one of the three complexes $F^{\bullet} \otimes_{R} N^{\bullet}, M^{\bullet} \otimes_{R} H^{\bullet}$, or $F^{\bullet} \otimes_{R} H^{\bullet}$ can be taken as representing the object $M^{\bullet} \otimes_{R}^{\mathbb{L}} N^{\bullet}$ in the semicoderived category $\mathrm{D}_{A}^{\text {sico }}(R$-mod $)$.

The left derived functors $\otimes_{R}^{\mathbb{L}}$ are well-defined by this construction according to [14, Lemma 2.7], whose conditions are satisfied by Lemma 6.4 and [16, Lemma 1.6]. To sum up this somewhat tedious construction, one can simply say that both the derived functors $M^{\bullet} \otimes_{R}^{\mathbb{L}} N^{\bullet}$ are computed by the total complex of the bar-tricomplex

$$
\cdots \longrightarrow M^{\bullet} \otimes_{A} R \otimes_{A} R \otimes_{A} N^{\bullet} \longrightarrow M^{\bullet} \otimes_{A} R \otimes_{A} N^{\bullet} \longrightarrow M^{\bullet} \otimes_{A} N^{\bullet}
$$

constructed by taking infinite direct sums along the diagonal hyperplanes.

Finally, we can return to the situation with a coherent commutative ring $A$ such that fp-injective $A$-modules have finite injective dimensions and a dualizing complex of $A$-modules $D^{\bullet}$ is chosen. As above, let $R$ be a flat commutative $A$-algebra and $T^{\bullet}=R \otimes_{A} D^{\bullet}$ be the corresponding relative dualizing complex. Given two complexes of $R$-modules $M^{\bullet}$ and $N^{\bullet}$, one replaces one or both of them with complexes of $A$-injective $R$-modules $I^{\bullet}$ and/or $J^{\bullet}$ endowed with morphisms of complexes of $R$-modules $M^{\bullet} \longrightarrow I^{\bullet}$ and $N^{\bullet} \longrightarrow J^{\bullet}$ with the cones coacyclic as complexes of $A$-modules. Then the object of the semicoderived category $M^{\bullet} \diamond_{T} \bullet N^{\bullet}$ can be computed in either of three equivalent ways as

$$
\begin{gathered}
D^{\bullet} \otimes_{A}\left(\operatorname{Hom}_{A}\left(D^{\bullet}, I^{\bullet}\right) \otimes_{R}^{\mathbb{L}} \operatorname{Hom}_{A}\left(D^{\bullet}, J^{\bullet}\right)\right) \\
\simeq \operatorname{Hom}_{A}\left(D^{\bullet}, I^{\bullet}\right) \otimes_{R}^{\mathbb{L}} N^{\bullet} \simeq \operatorname{Hom}_{A}\left(D^{\bullet}, J^{\bullet}\right) \otimes_{R}^{\mathbb{L}} M^{\bullet},
\end{gathered}
$$

where the isomorphism signs mean natural isomorphisms in the semicoderived category $\mathrm{D}_{A}^{\text {sico }}(R$-mod $)$. As the equivalence of categories $\mathrm{D}_{A}^{\text {sico }}\left(R-\bmod _{A-\mathrm{fl}}\right) \simeq \mathrm{D}_{A}^{\text {sico }}(R-\bmod )$ takes the one-term complex $R \in \mathrm{D}_{A}^{\text {sico }}\left(R-\bmod _{A-\mathrm{fl}}\right)$ to the relative dualizing complex $T^{\bullet} \in \mathrm{D}_{A}^{\text {sico }}(R-$ mod $)$, the relative dualizing complex $T^{\bullet}$ is the unit object of the tensor category structure $\diamond_{T} \bullet$ on the triangulated category $\mathrm{D}_{A}^{\text {sico }}(R-\bmod )$.

\section{REFERENCES}

[1] H. Becker. Models for singularity categories. Advances in Math. 254, p. 187-232, 2014. arXiv: 1205.4473 [math.CT]

[2] L. W. Christensen, A. Frankild, H. Holm. On Gorenstein projective, injective, and flat dimensions - A functorial description with applications. Journ. of Algebra 302, \#1, p. 231279, 2006. arXiv:math.AC/0403156

[3] J. Gillespie. Model structures on exact categories. Journ. of Pure and Applied Algebra 215, \#12, p. 2892-2902, 2011. arXiv:1009.3574 [math.AT]

[4] L. Gruson, C. U. Jensen. Dimensions cohomologiques reliées aux foncteurs $\varliminf^{(i)}$. P. Dubreil and M.-P. Malliavin Algebra Seminar, 33rd Year, Lecture Notes in Math. 867, 1981, p. 234-294.

[5] M. Hovey. Cotorsion pairs, model category structures, and representation theory. Math. Zeitschrift 241, \#3, p. 553-592, 2002. 
[6] P. Jørgensen. The homotopy category of complexes of projective modules. Advances in Math. 193, \#1, p. 223-232, 2005. arXiv:math.RA/0312088

[7] H. Krause. The stable derived category of a Noetherian scheme. Compositio Math. 141, \#5, p. 1128-1162, 2005. arXiv:math.AG/0403526

[8] S. Iyengar, H. Krause. Acyclicity versus total acyclicity for complexes over noetherian rings. Documenta Math. 11, p. 207-240, 2006.

[9] H. Krause. Approximations and adjoints in homotopy categories. Mathematische Annalen 353, \#3, p. 765-781, 2012. arXiv:1005.0209 [math.CT]

[10] L. Mao, N. Ding. Notes on FP-projective modules and $F P$-injective modules. Advances in Ring Theory, J. Chen, N. Ding, H. Marubayashi, Eds., Proceedings of the 4th China-Japan-Korea International Conference, June 2004, World Scientific, 2005, p. 151-166.

[11] B. Mitchell. The cohomological dimension of a directed set. Canadian Journ. of Math. 25, \#2, p. 233-238, 1973.

[12] J. Miyachi. Derived categories and Morita duality theory. Journ. of Pure and Appl. Algebra 128, \#2, p. 153-170, 1998.

[13] D. Murfet. The mock homotopy category of projectives and Grothendieck duality. Ph. D. Thesis, Australian National University, September 2007. Available from http://www.therisingsea. org/thesis.pdf .

[14] L. Positselski. Homological algebra of semimodules and semicontramodules: Semi-infinite homological algebra of associative algebraic structures. Appendix $\mathrm{C}$ in collaboration with D. Rumynin; Appendix D in collaboration with S. Arkhipov. Monografie Matematyczne vol. 70, Birkhäuser/Springer Basel, 2010. xxiv+349 pp. arXiv:0708.3398 [math.CT]

[15] R. Bezrukavnikov, L. Positselski. On semi-infinite cohomology of finite-dimensional graded algebras. Compositio Math. 146, \#2, p. 480-496, 2010. arXiv:0803.3252 [math.QA]

[16] L. Positselski. Two kinds of derived categories, Koszul duality, and comodule-contramodule correspondence. Memoirs of the American Math. Society 212, \#996, 2011. vi+133 pp. arXiv:0905.2621 [math.CT]

[17] A. I. Efimov, L. Positselski. Coherent analogues of matrix factorizations and relative singularity categories. Algebra and Number Theory 9, \#5, p. 1159-1292, 2015. arXiv:1102.0261 [math.CT]

[18] L. Positselski. Contraherent cosheaves. Electronic preprint arXiv:1209.2995v4 [math.CT] .

[19] L. Positselski. Dedualizing complexes and MGM duality. Journ. of Pure and Appl. Algebra 220, \#12, p. 3866-3909, 2016. arXiv:1503.05523 [math.CT]

[20] L. Positselski. Semi-infinite algebraic geometry. Slides of the presentation at the conference "Some Trends in Algebra 2015", Prague, September 2015. Available from http://www. karlin.mff.cuni.cz/ ^sta/pres/positselski.pdf or http://positselski.narod.ru/ semi-inf-nopause.pdf, or http://positselski.narod.ru/semi-inf.pdf.

[21] B. Stenström. Coherent rings and FP-injective modules. Journ. of the London Math. Society (2), 2, \#2, p. 323-329, 1970.

[22] J. Št'ovíček. On purity and applications to coderived and singularity categories. Electronic preprint arXiv:1412.1615 [math.CT] .

[23] P. C. Eklof, J. Trlifaj. How to make Ext vanish. Bulletin of the London Math. Society 33, \#1, p. $41-51,2001$.

[24] J. Trlifaj. Covers, envelopes, and cotorsion theories. Lecture notes for the workshop "Homological methods in module theory", Cortona, September 2000. 39 pp. Available from http://matematika.cuni.cz/dl/trlifaj/NALG077cortona.pdf

[25] A. Yekutieli. Dualizing complexes over noncommutative graded algebras. Journ. of Algebra 153, \#1, p. 41-84, 1992.

[26] A. Yekutieli, J. J. Zhang. Rings with Auslander dualizing complexes. Journ. of Algebra 213, \#1, p. 1-51, 1999. arXiv:math.RA/9804005 
[27] M. Porta, L. Shaul, A. Yekutieli. On the homology of completion and torsion. Algebras and Representation Theory 17, \#1, p. 31-67, 2014. arXiv:1010.4386 [math.AC]

Department of Mathematics, Faculty of Natural Sciences, University of Haifa, Mount Carmel, Haifa 31905, Israel; And

Laboratory of Algebraic Geometry, National Research University Higher School of ECONOMics, Moscow 117312; AND

Sector of Algebra and Number Theory, Institute for Information Transmission Problems, Moscow 127051, Russia

E-mail address: posic@mccme.ru 